DESIGN AND IMPLEMENTATION OF A HYBRID FACE RECOGNITION TECHNIQUE

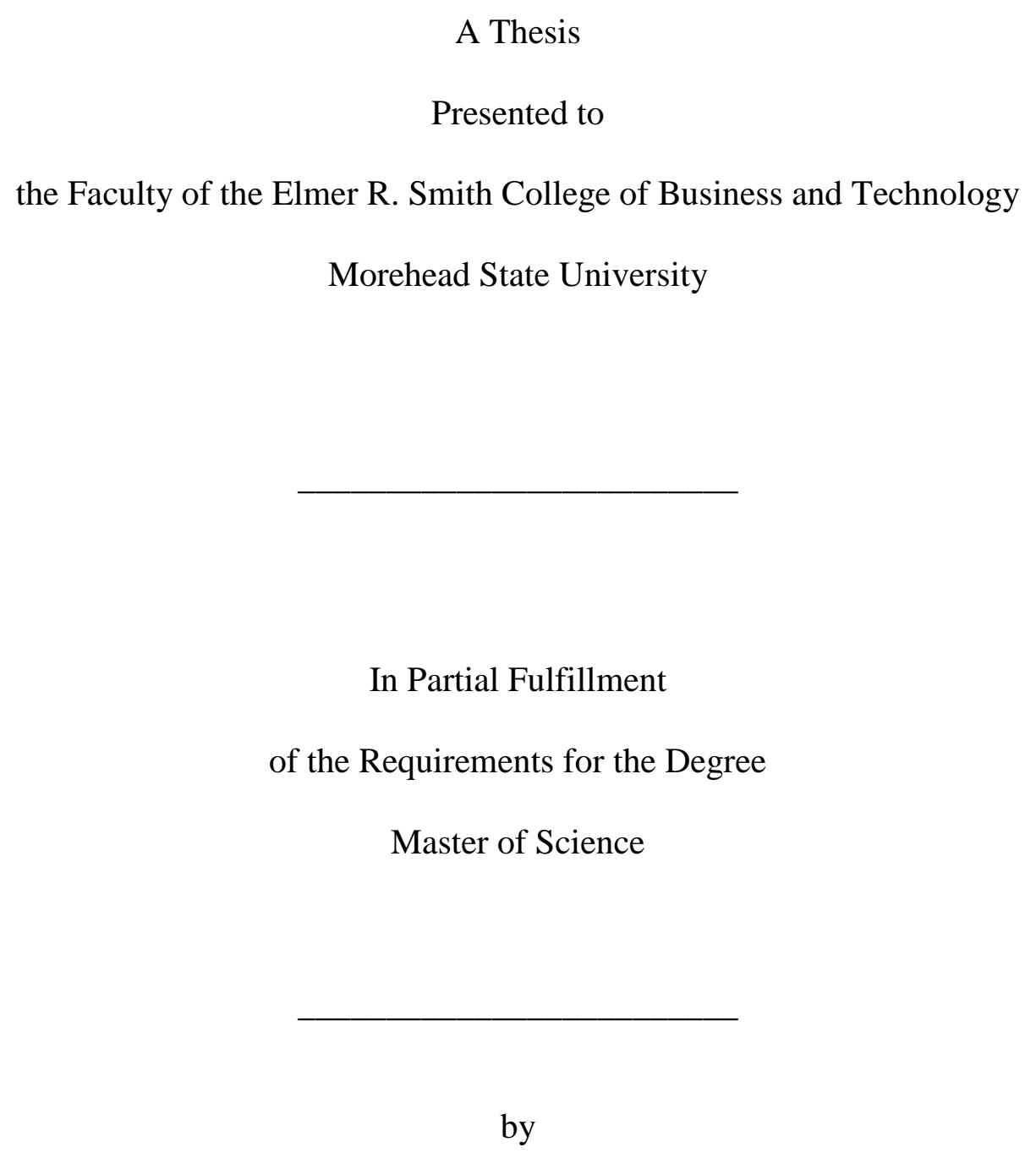

Asim S. Chaudhry

October 16, 2018 
ProQuest Number: 10980617

All rights reserved

INFORMATION TO ALL USERS

The quality of this reproduction is dependent upon the quality of the copy submitted.

In the unlikely event that the author did not send a complete manuscript and there are missing pages, these will be noted. Also, if material had to be removed, a note will indicate the deletion.

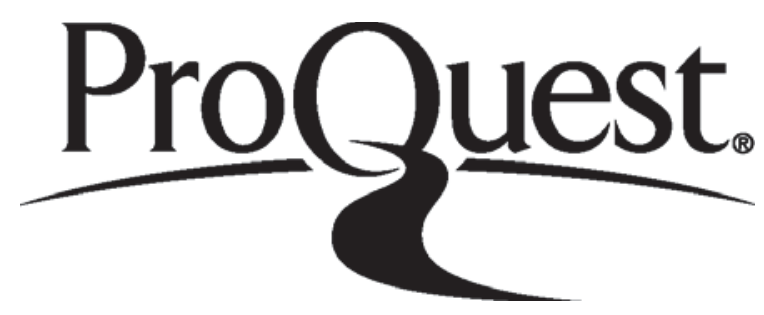

ProQuest 10980617

Published by ProQuest LLC (2018). Copyright of the Dissertation is held by the Author.

All rights reserved.

This work is protected against unauthorized copying under Title 17, United States Code Microform Edition (C) ProQuest LLC.

ProQuest LLC.

789 East Eisenhower Parkway

P.O. Box 1346

Ann Arbor, Ml $48106-1346$ 
Accepted by the faculty of the Elmer R. Smith College of Business and Technology, Morehead State University, in partial fulfillment of the requirements for the Master of Science degree.

Dr. Heba Elgazzar

Director of Thesis

Master's Committee:

, Chair

Dr. Ahmad Zargari

Dr. Sam Nataraj

Dr. Cheng Cheng

Date 


\section{DESIGN AND IMPLEMENTATION OF A HYBRID FACE RECOGNITION TECHNIQUE}

Asim S. Chaudhry

Morehead State University, 2018

Director of Thesis:

\section{Dr. Heba Elgazzar}

The problem of face recognition is a subject that has been researched for many years. Face recognition technology can be utilized in many different industries, it isn't solely designated for computer science relate fields. The purpose of this thesis is to continue this research and attempt to provide a powerful classifier that works on par with the algorithms currently on the market. This new algorithm will be known as the EFL Hybrid algorithm. This classifier is created by combining many popular algorithms. There are three major algorithms that will be explored in this thesis: Eigenfaces, Fisherfaces, and Local Binary Patterns Histograms. The Eigenfaces algorithm utilizes Principal Component Analysis (PCA) to project the face data to a low dimensional subspace where the data is distinct, and variation is maximized. These principal components are called the eigenvectors or Eigenfaces. The Fisherfaces algorithm employs Linear Discriminant Analysis (LDA) to reduce the dimensionality of the data. LDA projects the data such that in between class and within class scatter is maximized. The Local Binary Patterns 
Histogram (LBPH) is a classifier that uses texture descriptions based on local binary patterns (LBP). A new image can be created by taking the LBP from each pixel in the original image. This new LBP image is split up into blocks, each block is a histogram. These three algorithms are tested against images provided by the Yale Face Database (Yale Face Database, n.d.). The experimental results show that both the LBPH and Fisherfaces classifiers perform better than the Eigenfaces classifier. Combining these three algorithms can provide the highest accuracy, as long as the LBPH is ranked at the highest.

Accepted by: , Chair

Dr. Ahmad Zargari

Dr. Sam Nataraj

Dr. Cheng Cheng 


\section{TABLE OF CONTENTS}

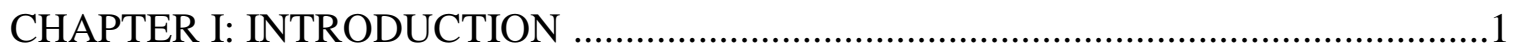

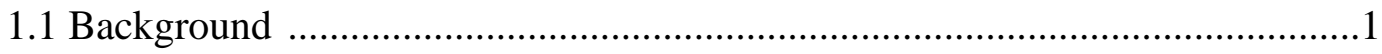

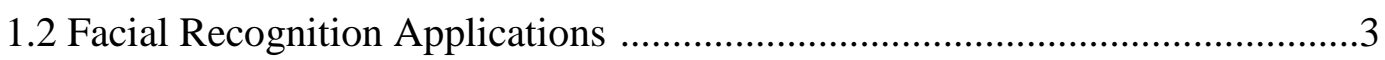

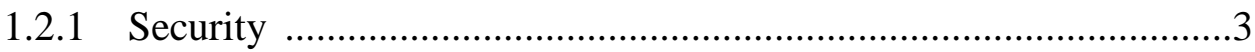

1.2.2 Law Enforcement ...............................................................

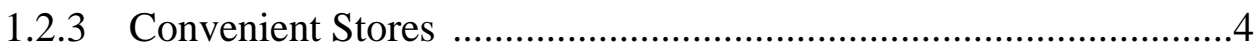

1.2.4 Attendance ...........................................................................

1.2.5 Identity Documents ................................................................

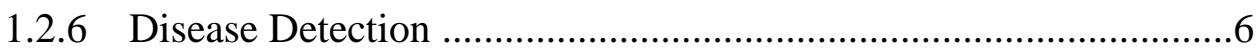

1.2.7 Assisting the Visually Impaired .................................................

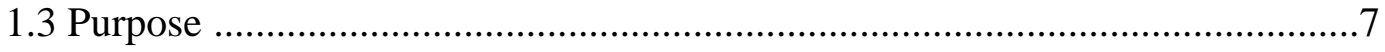

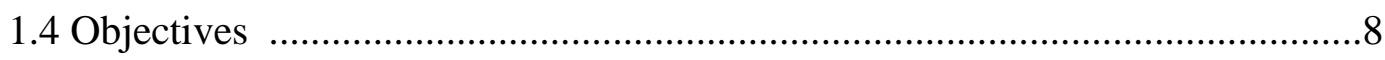

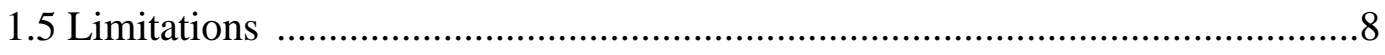

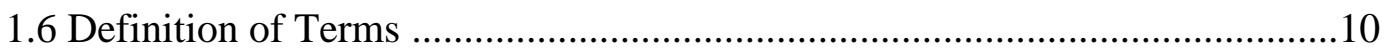

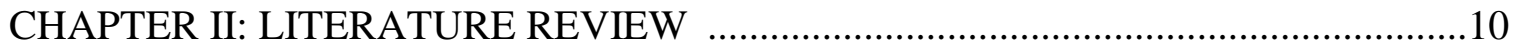

2.1 Eigenfaces using PCA …………….......................................................10

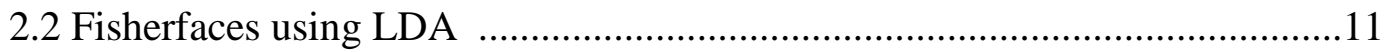

2.3 Local Binary Patterns Histograms (LBPH) ………………........................13

2.4 Scale-Invariant Feature Transform (SIFT) ………….................................15

2.5 Speed-Up Robust Feature (SURF) …………………………....................15

2.6 Elastic Bunch Graph Matching …………………….............................16 


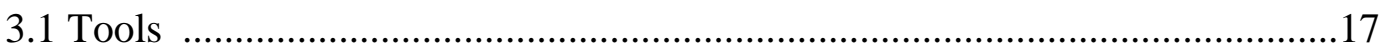

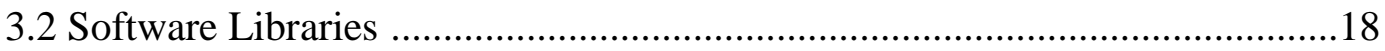

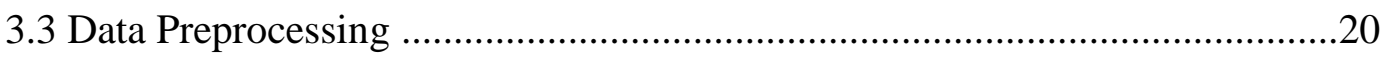

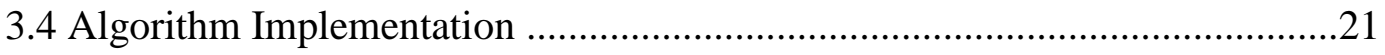

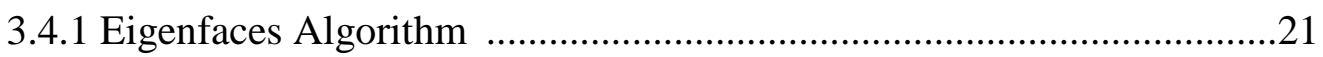

3.4.2 Fisherfaces Algorithm ...................................................................24

3.4.3 Local Binary Patterns Histogram Algorithm .......................................... 26

3.4.4 EFL Hybrid Algorithm ...................................................................28

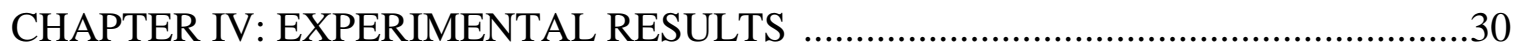

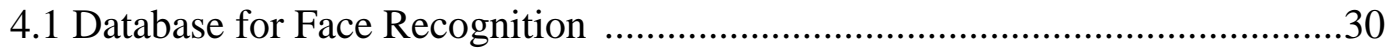

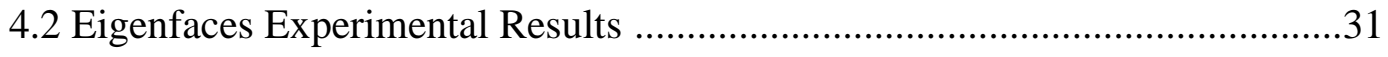

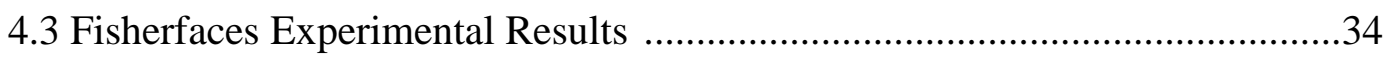

4.4 Local Binary Patterns Histograms Experimental Results ................................37

4.4 EFL Hybrid Algorithm Experimental Results .................................................39

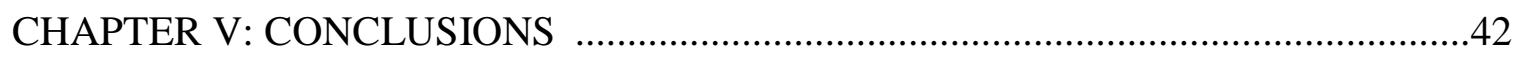

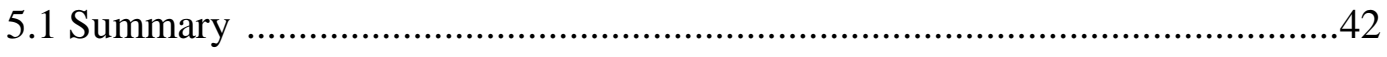

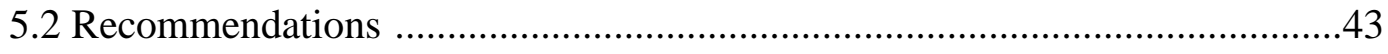

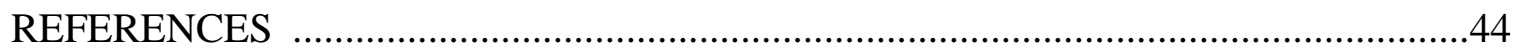


Appendix A: Face Recognition Parent Class (Part 1) ........................................48

Appendix B: Face Recognition Parent Class (Part 2) ........................................49

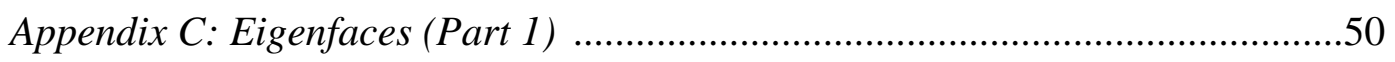

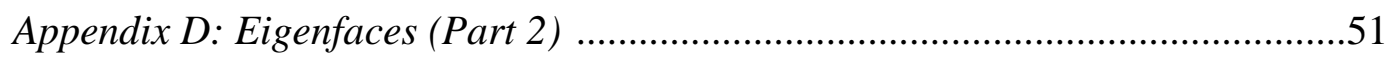

Appendix E: Fisherfaces (Part 1) ……………………...............................52

Appendix F: Fisherfaces (Part 2) ...............................................................

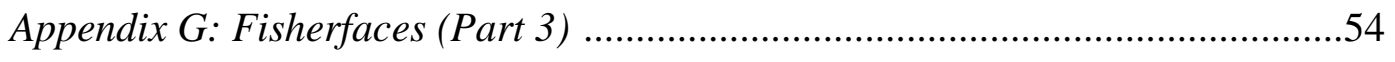

Appendix H: Local Binary Patterns Histograms (Part 1) ....................................55

Appendix I: Local Binary Patterns Histograms (Part 2) ....................................56

Appendix J: Local Binary Patterns Histograms (Part 3) ....................................57

Appendix K: Local Binary Patterns Histograms (Part 4) .................................58

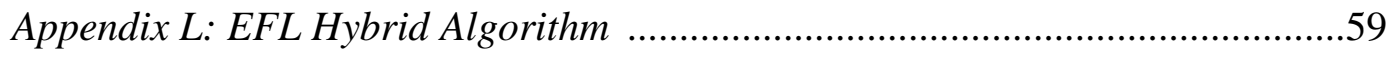




\section{CHAPTER I: INTRODUCTION}

\subsection{Background}

Facial recognition is a technology that has been evolving steadily over the last decade. A skill that is developed by children at a very young age has taken engineers years to develop. A child will naturally develop his sight; however, a computer must be taught how to see. Giving a computer the ability of sight is where the science of Computer Vision began. Gary Bradski describes computer vision as, "the transformation of data from a still or video camera into either a decision or a new representation" (Bradski, 2015, p. 18). An image by itself isn’t something that a computer can comprehend, it needs to be translated to a different form. Everything a computer understands is represented as a number, more specifically a binary number. A machine tends to be most comfortable with numbers, so transforming the image into a group of numbers is the most logical way to go.

Figure 1 shows how an image can be represented to allow a machine to understand it. Images can be viewed as a matrix of numbers with each number representing a pixel in the image. In terms of black and white images, these pixel numbers will represent the shades of gray of the image. For a color picture each pixel is given three values. These three numbers represent the level of red, green, and blue. The values range from 0 to 255 providing many different color possibilities. Once the images are in number form they can be manipulated using tools such as Machine Learning to help give machines the ability to perceive objects. 


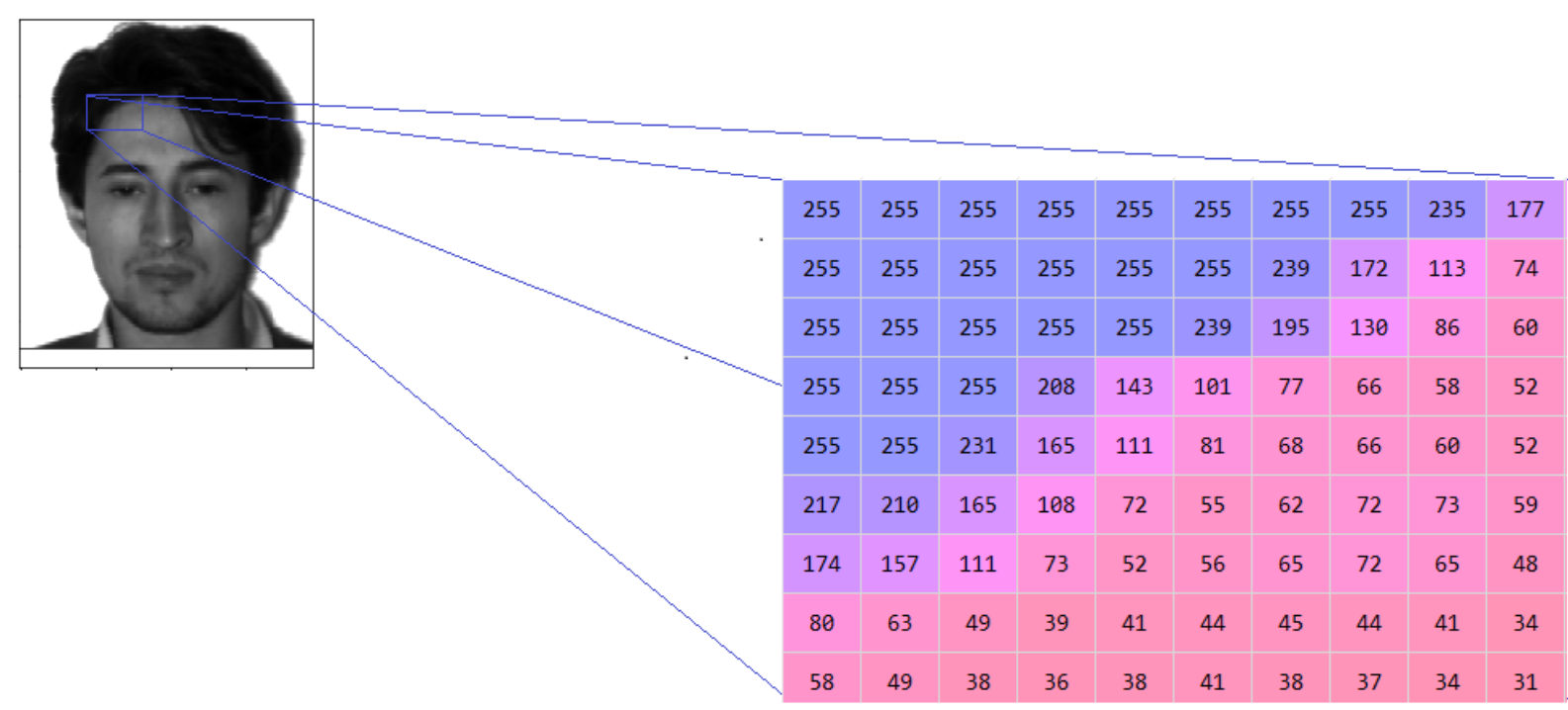

Figure 1: Image Representation in a Computer (Yale Face Database, n.d.)

Machine Learning is a programming implementation which allows a machine to learn a specific pattern rather than having the pattern explicitly programmed into it. Mehmed Kantardzic further explains that, "[Machine Learning] algorithms learn by searching through an ndimensional space of a given data set to find an acceptable generalization" (Kantardzic, 2011, p. 89). A computer can perform actions more rapidly and efficiently that a human being. It would take a computer a fraction of the time to model complex data than it would for a human. It may also find patterns that it's human counterpart may never have discovered. There are two important methods in Machine Learning: supervised learning and unsupervised learning. In a supervised learning environment, the programmer provides data that serves as both the input and the expected output. The machine would then perform its calculations on the data and obtain an output. This output can then be compared to the expected output. What the programmer is doing is teaching the machine based on examples. On the other hand, the unsupervised learning method 
doesn't rely on having an expected output. It is the machine's responsibility to see how the data fits together. Both of these methods can be effective depending on the requirements of the project.

\subsection{Facial Recognition Applications}

Before the inner workings of face recognition technology can be discussed, it is important to have an understanding as to why this technology is important. The reality is the use of face recognition applications isn't only isolated to the computer science industry. Face recognition can provide many useful applications to all kinds of industries, from law enforcement to the educational system. More specific applications will be discussed in this section.

\subsubsection{Security}

Face recognition technology can be used as another form of authentication when attempting to access personal accounts. Whether that be personal computers, banking accounts, or even email accounts having an extra layer of security can be invaluable when a person's livelihood is on the line. Automated Teller Machines (ATMs) already have cameras installed to help prevent tampering. This camera can instead be used as a form of identification, only allowing the user to access their account if their face is in the system. Securing this data behind a biometric security system, such as face recognition, can be vital. Facial recognition is already being used as a form of access control. Jeffrey S. Coffin developed a security method that uses face recognition to identify individuals in order to determine their clearance access (Coffin, 1999). In the media, biometric security measures are generally boiled down to retinal scans, and finger print scans. Facial recognition is a very powerful biometric security system that can improve any access system. 


\subsubsection{Law Enforcement}

Law Enforcement is another industry that can benefit from facial recognition software. One of the jobs required of policemen is tracking down criminals. Face recognition software can automate this process by automatically tracking these criminals without needing any manpower besides making the arrest. Cameras are set up all around the country, from traffic cameras, street cameras, to private security cameras. The law enforcement officials can feed their database of criminals into a facial recognition algorithm and use these cameras to track criminals all around the world. Writing speeding tickets is another job police officers bog their time down with. Sensors can be set up to check whether the car is going too fast. Then cameras can recognize the face and automatically send a speeding ticket. Setting up cameras and speeding sensors may not be very cost effective now; however, as technology progresses the cost of these things continue to lower.

\subsubsection{Convenient Stores}

The police aren't the only group that needs to track users. Convenient stores can even use this technology to track customers throughout their stores. There are stores already employing this technology, such as seven-eleven. Seven-eleven has applied a facial recognition system to 11,000 stores across Thailand allowing them to collect useful data by utilizing these functions (Chan, 2018):

- identifying loyalty members

- analyzing in-store traffic

- monitoring product levels

- $\quad$ suggesting products to customers 
- measuring the emotions of customers

Data is at a premium right now, companies such as Facebook are doing whatever they can to collect as much data as possible from their users. In such a data driven society, this kind of technology can bring a lot of growth to a company. With all this information being collected from the customers, attempted thefts can be as trivial as looking through the cameras to see who entered the store. Law enforcement can also provide these convenient stores with their criminal database, so that the system can automatically alert the police if it spots a threat.

\subsubsection{Attendance}

The educational systems have some processes that can be improved by employing facial recognition technology. Taking attendance is an important part of class time to ensure that all students are present; however, the larger the class the more difficult and longer it takes to check. The problem is compounded in classes that fill entire auditoriums. These systems, though not used often, already exist. Abhishek Jha developed an automated attendance system that can invaluable in the education industry (Jha, 2018). This system can help cut down the amount of time needed in class by allowing the professor to jump straight into the material rather than waste time calling everyone's name one at a time. Many universities have already forgone the use of attendance because sometimes it just isn't feasible with the number of students they receive. By automating this process, the university can track which students are attending lectures. 


\subsubsection{Identity Documents}

Identity theft is a very serious issue that is prevalent in society. Adding another form of security to identity documents can help prevent this kind of theft. Carlos Cobian Schroeder developed a biometric security procedure that uses face recognition to combat identity theft (Schroeder, 1998). All kinds of identity documents, such as passports and visas, can be backed up by identity data which includes the person's face. Computers are then set up to scan the person's face against the face in the system to authenticate the user. Faces can be unique, so it would be difficult for a thief to gain access to an identity. There are reports that the Australian Passport Office is creating a face recognition system. The system is designed to be used in online government services, enabling the user to easily provide authentication (Lee, 2017). This is a first step at employing this technology into the ecosystem, if it proves successful then it may spread to other parts of the industry.

\subsubsection{Disease Detection}

Disease detection isn't something most people would associate with facial recognition; however, it is something that can be vastly improved by employing this technology. Paul Kruszka and his colleagues developed a system that uses facial analysis technology to help assist in early detection of the disease know as 22q11.2 deletion syndrome (Kruszka, 2017). This disease is defined as a common microdeletion syndrome that is characterized by "congenital heart disease, immunodeficiency, hypoparathyroidism, palatal, gastrointestinal, skeletal, and renal abnormalities, characteristics facial features, developmental and speech delay and an increased risk for psychiatric illness" (Kruszka, 2017, p.2). Early detection of this disease can help the victims immensely. 


\subsubsection{Assisting the Visually Impaired}

Face recognition software can be used to help blind people see. Of course, it won't let them literally see, but it can help them recognize people. It can be troublesome for a visually impaired individual to recognize whomever they are currently speaking with in social settings. By applying face recognition technology to this situation, the visually impaired can have more enjoyable social interactions. This face recognition system can work by using a smart phone's built in camera. The phone would wirelessly transmit the face data to a remote server that performs the recognition, and then transmits it back to the phone (Jafri, 2013). The phone can then provide a sound queue based on the individual it classified, or even say the person's name.

\subsection{Purpose}

Due to the increasing popularity of face recognition algorithms, it is important to have a deeper understanding of how these algorithms work. This thesis will outline the process of creating a facial recognition algorithm. Face recognition algorithms have been developed and optimized for many years, it isn't a new technology. There are many popular algorithms that currently exist. The purpose of this thesis is to explore current algorithms and propose a hybrid algorithm to improve the overall performance and provide better experimental results. The focus of this study will be on three algorithms and these algorithms will be compared and analyzed. A new hybrid algorithm is proposed that is based on the combination of these specific algorithms to provide better experimental results. 


\subsection{Objectives}

There are three main objectives for the proposed work in this thesis:

1. Implementing three facial recognition algorithms: Eigenfaces, Fisherfaces, and Local Binary Patterns Histograms

2. Testing and analyzing the experimental results of these three algorithms

3. The creation of a new hybrid facial recognition algorithm that combines three algorithms

\subsection{Limitations}

Training a machine learning algorithm can take a great deal of data to achieve maximum optimization and reduce error. Depending on the algorithm employed, these "learning" sessions can take a very long time. The training sessions can put a great deal of stress on the computer, so its imperative that a powerful computer is used. A computer with a stronger processor can handle more calculations and operations in a lower amount of time. In the case of image processing, a powerful graphics card can also be useful. The algorithms being explored in this project can take some time; however, the dataset is small enough that training times could be lessened. For this project, a high-quality computer is being used. 


\subsection{Definition of Terms}

The following are definitions of terms that will be used extensively within this thesis.

Principal Component Analysis (PCA): Transformation of the dataset to a lower dimensional set where most of the important information still exists (Kantardzic, 2011).

Linear Discriminant Analysis (LDA): A tool used to construct a discriminant function providing different results when applying data from different classes. (Kantardzic, 2011).

Local Binary Patterns: A texture description operator that assigns a label to every pixel of an image by thresholding the neighborhood of the pixel with the center value (Ahonen, 2006).

Training Set: The data set that is reserved for training the classifier.

Testing Set: The data set that is reserved for testing the results of a fully trained classifier.

Confusion Matrix: A table that displays in-depth results of the algorithm's performance by comparing the predicted class to the actual class. 


\section{CHAPTER II: LITERATURE REVIEW}

\subsection{Eigenfaces using PCA}

The first algorithm that will be implemented is the Eigenfaces classifier. For the purpose of this thesis. it is important to discover what research has already been completed on this subject. Eigenfaces is an algorithm that uses Principal Component Analysis. PCA is a data reduction tool used for multivariate data. Kantardzic defines PCA as, "a method of transforming the initial data set represented by vector samples into a new set of vector samples with derived dimensions" (Kantardzic, 2011, p. 70-71). The theory behind using PCA is that the data is projected to a subspace where there is maximum variance. This tool facilitates the transformation of a larger data set into a smaller more manageable set which contains all the essential parts to reduce the dimensionality. PCA is an unsupervised method, meaning it doesn't require class information (Rahman, 2012). Features, or eigenvectors, are extracted from a covariance matrix, a matrix that holds the covariance for each data element.

Matthew Turk and Alex Pentland employed Principal Component Analysis in the development of an algorithm called Eigenfaces (Turk, 1991). After applying PCA to the dataset, they obtained the eigenvectors. Since vectors are in the form of $1 \mathrm{xN}$, they need to be reformatted before they can be viewed as Eigenfaces. Turk and Pentland found that you can represent an individual face as a linear combination of Eigenfaces (Turk, 1991). Each Eigenface would be multiplied by a constant or weight, and then the resulting values are summed up. This algorithm was tested on a database with over 2500 images, 16 subjects, and each image had a resolution of 512x512. Turk and Pentland obtained an accuracy of 96\% correct over lighting variation, $85 \%$ correct over orientation variation, and 64\% correct over size variation (Turk, 1991). 
Another form of PCA exists as the Modular Principal Component Analysis algorithm. This method involves splitting the image into sub images. After these images are split up, the PCA steps are applied. These sub images are placed into a training set filled with other sub images. A covariance matrix can then be computed from this new set of data, from which the eigenvectors can be extracted. Ambika, Arathy and Srinivasa completed an experiment using MPCA on their face data (D. Ambika, 2012). They tested this algorithm against three different face databases: the ORL, JAFFE, and Indian databases. The ORL database provided them 40 distinct subjects, and the classifier had an accuracy of $99 \%$ (D. Ambika, 2012). The Indian database also had 40 distinct subjects, giving an accuracy of 99\% (D. Ambika, 2012). Finally, the JAFFE database, providing 10 distinct subjects, had an accuracy of 99\% (D. Ambika, 2012). This is clearly a strong algorithm for face recognition.

\subsection{Fisherfaces using LDA}

The Fisherfaces algorithm differs from Eigenfaces in that it uses Linear Discriminant Analysis. LDA involves "finding a set of weight values that maximizes the ratio of the betweenclass to the within-class variance of the discriminant score for a preclassified set of samples" (Kantardzic, 2011, p. 163). LDA is a classification model that was originally created by R. A. Fisher in the article The Use of Multiple Measurements in Taxonomic Problems (Fisher, 1936). Despite having its start in plant classification, this tool has applications in facial recognition. LDA puts a bigger emphasis on the classes in the data. When the data is trained, the LDA ensures that the separation in between different classes is maximized.

Peter N. Belhumeur explores the use of LDA in his Fisherfaces algorithm by comparing its effectiveness to the Eigenfaces algorithm (Belhumeur, 1997). The Fisherface algorithm starts with obtaining the between-class and within-class scatters for the data. A projection is created 
from this data that maximizes the ratio of the two scatters (Belhumeur, 1997). From this projection the eigenvectors are obtained. These vectors can then be reformatted into Fisherfaces. Belhumeur compared the results from his Eigenfaces algorithm which obtained an error rate of $24.4 \%$ to the results of his Fisherface algorithm, which obtained an error rate of $7.3 \%$ (Belhumeur, 1997). An error rate of $7.3 \%$ is very impressive for a face recognition algorithm.

Another Fisherface experiment was completed by Mustamin Anggo and La Arapu (Anggo, 2018). Following the algorithm proposed by Belhumeur, they created their own version of the Fisherface classifier. This version followed the original structure of the Fisherface algorithm very closely. After running the algorithm through their dataset, they arrived at an accuracy of 93\% (Anggo, 2018). This experiment provided an additional source of results from a different face database.

Wenyi Zhao, in his paper, attempted to use only LDA in face recognition (Zhao 1998). He found that this method doesn't function properly. There are three cases where a pure LDA face recognition classifier fails (Zhao 1998):

- The testing samples are from classes not present in the training set

- The testing samples are different from the data in the training set

- The samples have different backgrounds

He managed to solve this issue by employing PCA alongside LDA. He completed his experiment on the FERET face database. The database contained 1316 training images from 526 classes and 298 testing images from 298 different classes. An accuracy of 95\% was obtained using this classifier (Zhao 1998). 


\subsection{Local Binary Patterns Histograms (LBPH)}

Local Binary Patterns Histograms is a very simple and popular algorithm in facial analysis. The LBP operator is highly discriminative. It's invariance to monotonic gray-level changes and efficiency allow it to be a powerful tool in facial analysis (Ahonen, 2006). A face can consist of many of patterns that can make the LBP texture features invaluable in facial classification. LBP can be used to describe both the texture and the shape of an image (Rahim, 2013). Timo Ahonen and Matti Pietikainen present an experiment where they attempt to use local binary patterns in facial recognition (Ahonen, 2006). Each pixel in the face image is given a new value based on the values of the pixels in its 'neighborhood'. Ahonen and Pietikainen define the neighborhood as a $3 \times 3$ matrix around the original pixel (Ahonen, 2006). Once this LBP image is created it is further subdivided into regions. These regions are then concatenated together into a large histogram. This algorithm was run on the FERET database, which contains 14,051 gray-scale images or 1,199 individuals and obtained an accuracy of $97 \%$.

Abdur Rahim and his colleagues also implemented the Local Binary Patterns algorithm (Rahim, 2013). They used LBP for face recognition by splitting it into three different parts: face representation, feature extraction, and classification (Rahim, 2013). The face is first represented as an LBP image, then the histograms are extracted. The classification phase is determined by comparing histograms. Rahim explores three different methods of comparing histograms: Histogram Intersection, Log-likelihood Statistic, and Chi square statistic (Rahim, 2013). The image dataset contains images taken from either a camera or a face database. The classifier is fed a total of 2000 total faces. 1980 of these faces are recognized, while 20 are unrecognized, yielding an accuracy of 99\% (Rahim, 2013). 
There is a variation of this algorithm called the Local Gabor Binary Pattern Histogram Sequence. This classifier is robust to variation of imaging conditions and has more discrimination power (Zhang, 2005). The combination of Gabor filters and Local Binary Patterns enhances the algorithm. Wenchao Zhang created a classifier using this method (Zhang, 2005). He argues that the generalization obtained when training some of the popular algorithms is a problem. He hopes to avoid these issues by completely forgoing the training stage. His algorithm works in several steps (Zhang, 2005):

1. The input face is normalized.

2. Using Gabor filters, several Gabor Magnitude Pictures are obtained.

3. The Gabor Magnitude Pictures are converted to Local Gabor Binary Pattern images

4. The LGBP is subdivided into rectangle regions

5. Histograms are concatenated to form a total histogram

Zhang uses two approach to compare the histograms: direct matching and weighted matching (Zhang, 2005). The direct matching method simply compares the histograms; on the other hand, weighted matching applies a weight to regions of the face that are most important for recognition. Zhang ran his classifier on the FERET face database and obtained an accuracy of 94\% using the direct method, and 98\% using the weighted method (Zhang, 2005). 


\subsection{Scale-Invariant Feature Transform (SIFT)}

The Scale-Invariant Feature Transform algorithm is a useful tool in detecting and describing feature points on an image. This algorithm was originally created for use in object detection; however, it has transitioned into facial analysis. SIFT can extract gray-level features using local patterns taken from a scale-space decomposition of the image (Bicego, 2006). As the name suggests the features that are extracted from this algorithm are invariant, or never changing, based on changes in the environment such as light and distortion making these very distinct features. Manuele Bicego implemented this algorithm by comparing the SIFT features of the input face to the training set (Bicego, 2006). He used three different techniques to identify the correct face. These techniques were: calculating the distance between all the features to find the minimum distance, matching the eyes and mouth features, and matching features based off their grid (Bicego, 2006).

\subsection{Speed-Up Robust Feature (SURF)}

The creation of the Speed-Up Robust Features method was influenced by SIFT. Like SIFT, this algorithm involves obtaining features that are invariant to changes in the environment. SURF involves two important phases: detection and description. In the detection phase, important points of interest are found on the image. In order to find these points of interest, blob-like structures are located where the determinant is at maximum (Du, 2009). The description phase describes the features using Haar Wavelets. 


\subsection{Elastic Bunch Graph Matching}

The Elastic Bunch Graph Matching algorithm uses face graphs to model the data. This classifier finds distinct local features of the face. It is inspired by biological processes in the brain. One of these is the Gabor wavelet. Gabor wavelets can be defined as, "biologically motivated convolution kernels in the shape of plane waves restricted by the Gaussian envelope function" (Wiskott 1997, p.2). This feature is found to be a good model of early visual processing in the brain (Wiskott 2014). The matching algorithm is also based on dynamic link matching, a model of invariant object recognition in the brain (Wiskott 2014). Feature jets are used to form a face graph. Jets can be defined as a small patch of gray values in an image around a given pixel (Wiskott 1997). The face recognition process involves measuring the similarity of the face graphs. Laurenz Wiskott utilized Elastic Bunch Graph Matching in her paper, using the ARPA/ARL FERET database, to obtain an accuracy of 98\% (Wiskott 1997). 


\section{CHAPTER III: METHODOLOGY}

This section will present the methods for achieving the objectives presented in the introduction of this thesis. The first objective is to implement three face recognition algorithms. The procedure of implementing these algorithms is discussed in this chapter, as well as the software libraries that will assist in programming the classifiers. The second object involves the testing and analyzing of the three algorithms. Properly preprocessing the data is important so that it can provide the best experimental results when employed in the algorithms. Tools and software libraries are provided that will create tables and plots to help analyze the potential findings. The last objective is to create a hybrid algorithm that combines the three algorithms that are already implemented. The implementation of this EFL hybrid algorithm will be discussed in the final section.

\subsection{Tools}

The face recognition algorithms implemented for this thesis are coded in their entirety using Python. Python is a very useful language for applications in data science and machine learning. It is very easy to use, while providing quite a bit of complexity. It also provides useful tools for computer vision and machine learning through its many libraries which are included in the Anaconda distribution. The Anaconda Python distribution is a platform that allows organizations to develop machine learning and data science related projects (Anaconda, 2018). Anaconda also provides as robust programming development environment called Spyder. Combined with the Anaconda tools and Spyder editor all the tools necessary to complete this project are provided for. Training this algorithm may take a lot of time depending on how powerful of a machine is used. Fortunately, this project will run on a laptop which contains an 
Intel® Core $^{\mathrm{TM}}$ i7-6700HQ processor accompanied by an NVIDIA GeForce GTX 1070 graphics card. These tools will be integral to implementing the algorithms stated in the objectives.

\subsection{Software Libraries}

Python has access to many powerful libraries that will be imperative in the completion of this project. One of these libraries is OpenCV. The Open Source Computer Vision (OpenCV) library was created to provide a simple computer vision infrastructure that can help build complex applications rapidly (Bradski, 2015). This library makes it easier and more convenient to create powerful application in the computer vision domain. OpenCV provides over 500 useful functions for many different areas of computer vision including (Bradski, 2015):

- Factory product inspection

- Medical imaging

- Security

- User interface

- Camera calibration

- Stereo vision

- Robotics

This library also provides invaluable functions to help with the creation of facial recognition algorithms. For example, OpenCV comes equipped with a function that performs principal component analysis on the data. This provides an easy method to extract eigenvectors from a set of data. OpenCV was originally written in $\mathrm{C}$ and $\mathrm{C}++$; however, they do maintain development and maintenance on an interface for Python. OpenCV will provide shortcuts in the programming that can help reduce the amount of coding time. 
Another useful Python library that will be utilized is NumPy. This library provides a powerful tool, the NumPy array, that is practically a standard in Python related data science. The NumPy array can be described as a multidimensional, uniform collection of elements (Walt, 2011). These arrays can have any number of dimensions and a combination of many different data types such as integers, Booleans, and strings (Walt, 2011). NumPy also contains many useful functions for manipulating matrices. Some of these functions include: inverting matrices, sorting vectors, and transposing vectors. These functions will be vital since the face data will be stored in matrices.

The ability to view the images and craft charts is something that is invaluable in this project. The matplotlib library provides tools to achieve these goals. Matplotlib is a python library designed to help create plots of the data. This library was created to emulate graphics commands that are already available in MATLAB ${ }^{\mathrm{TM}}$ (Hunter, 2007). This library complements greatly with NumPy which provides access to highly powerful arrays. The data employed in this thesis is almost entirely full of images. This library provides a useful tool to view these images and the transformations applied on them.

Another powerful library used for machine learning is the scikit-learn library. This library provides powerful tools for classification, regression, and clustering. While these tools are useful, for the most part the face recognition program implemented in the thesis was coded from scratch. The PCACompute() method from OpenCV is one of the exceptions. The scikit-learn library is also useful because it provides excellent functions for testing algorithms. Its use in this experiment is restricted to the use of the confusion matrix method. This method allows easy creation of confusion matrices which are valuable in analyzing the experimental results. 


\subsection{Data Preprocessing}

Before the data can be fed into a recognition algorithm, it must first be processed into the proper form. Each specific algorithm has their own form of data processing; however, there are some general transformations that can be completed for all of them. As stated previously, images are stored as matrices where each entry represents a pixel. Each pixel contains three values which can represent all the colors in the spectrum. The values are formatted as red, green, blue $(\mathrm{rgb})$. For the purposes of this algorithm, the actual color of the image is unimportant. The algorithms used in this project will focus more on the color intensity rather than the actual colors. To get the most out of the algorithms, grayscale images are used. A grayscale image represents the data in shades of gray. Instead of having three values (rgb) for each pixel in the image matrix, there is only one value which represents intensity of the gray. The database being used in the project already has the images formatted as grayscale simplifying the data preprocessing steps. The last step is to split the data into training and testing sets. The training set is what will be used to train the algorithms. It is important to have many examples for every class in the training set. The more data in the training set, the more accurate the classifier becomes. The testing set will have the data that will be tested against the trained algorithm to determine its accuracy. For this project, a 4:1 ratio of training to testing will be used to split up the data. It is important to make sure that there is at least one instance of each class in the testing sample otherwise it would be impossible to test all the classes. 


\subsection{Algorithm Implementation}

One of the objectives is to implement three face recognition algorithms. In this section, the implementations of each algorithm will be discussed. This thesis will explain the implementation of three major face recognition algorithms: Eigenfaces, Fisherfaces and Local Binary Pattern Histograms. These three algorithms will serve as the structure for the hybrid algorithm which will provide improved experimental results over each of these components.

\subsubsection{Eigenfaces Algorithm}

Training an Eigenfaces algorithm involves obtaining the eigenvectors of the dataset, by employing Principal Component Analysis, then calculating the weights for each face to perform the recognition process. To perform PCA on the data, it must first be transformed into a vector format. Given of a set of images $X=\left\{X_{1}, X_{2}, \ldots, X_{k}\right\}$ of dimensions NxM, the images must be transformed to a vector form of $1 \mathrm{x}\left(\mathrm{N}^{*} \mathrm{M}\right)$ (Turk, 1991). From this an entirely new table is created where each row represents a face. At this point the data is ready to perform principal component analysis upon. Its important to remove all the common features of the faces so that all the unique features can remain (Turk, 1991). To achieve this, the mean face $\Psi$ can be calculated using the equation in by adding up all the face vectors $\mathrm{X}_{\mathrm{i}}$ and dividing by the total number of faces $\mathrm{k}(1)$.

$$
\begin{aligned}
& \Psi=\frac{1}{k} \sum_{i=1}^{k} X_{i} \\
& \Phi=X i-\Psi
\end{aligned}
$$

Each face is subtracted by the mean face leaving only the unique data as seen in the equation, where $\Phi$ is the normalized face vector (2). Figure 2 displays what the average face 
would look like. This face should look like a generic person with very little unique features. Subtracting this face from the rest of the data set will in theory leave only distinct features.

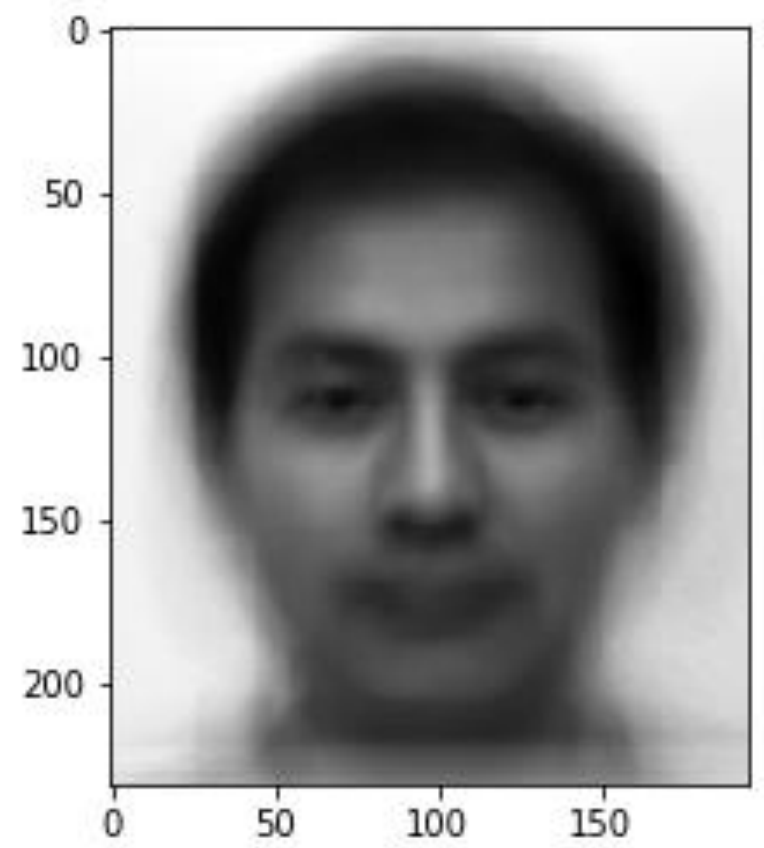

Figure 2: Average Face of Training Set (Yale Face Database, n.d.)

From here the covariance matrix is ready to be computed. The covariance matrix can be calculated by using equation (3), where $\mathrm{C}$ is the covariance matrix and $\Phi$ is the normalized face vector . To multiply two vectors, one of them must first be transposed T. Eigenvectors will be extracted from the covariance matrix. The eigenvectors are sorted by their eigenvalues, then the top X number of components are extracted (Turk, 1991). Generally having a small number of eigenfaces isn't preferable.

$$
C=\frac{1}{k} \sum \Phi \Phi^{\mathrm{T}}
$$

OpenCV comes equipped with a function, called PCACompute(), which will complete the PCA task and provide the user with the mean face and the eigenvectors. These eigenvectors 
can be reshaped into their original forms and be viewed as a face. Each face in the training set can be portrayed as a linear combination of these eigenfaces. Setting the number of components from the PCA method to 15 will yield 15 eigenfaces. These 15 faces contain all the important distinct features from the entire dataset. By applying a weight to each of these eigenfaces, any of the faces in the training set can be recreated. At this point, weights are calculated for each face in the dataset. Once all the weights are calculated, the algorithm is ready to start classifying faces.

The face recognition task is very similar to the training process. The input image must first be flattened so that it fits the form of $1 x\left(N^{*} M\right)$. Next the mean face $\Psi$ is subtracted from this input vector to ensure that only unique features remain. The weights are then calculated for the face, so that they can be portrayed as a linear combination of the Eigenfaces. Using Euclidean distance, these weights are compared to the weights in the training set. The face with the lowest Euclidean distance is deemed to be the same class as the input face. This algorithm can be summed up by the flow chart provided in Figure 3.

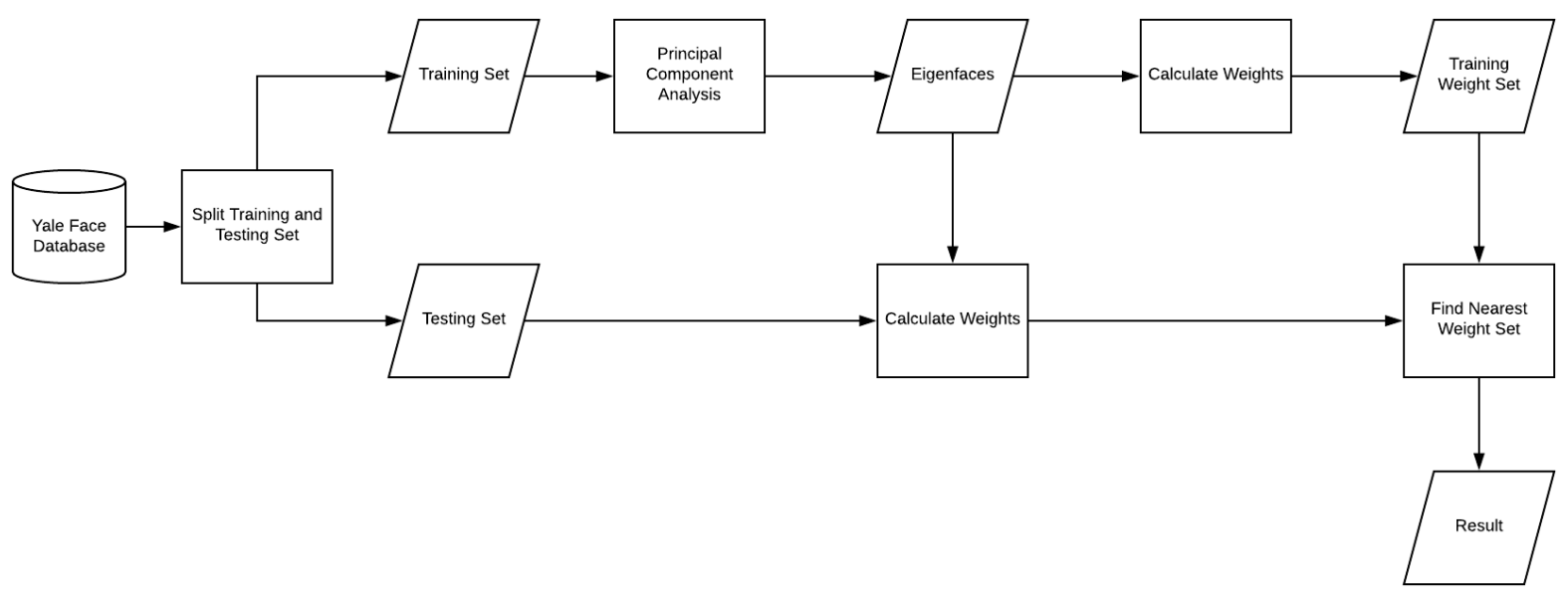

Figure 3: Eigenfaces Flow Chart 


\subsubsection{Fisherfaces Algorithm}

The Fisherfaces algorithm was developed as an improvement to the Eigenfaces algorithm. This method uses Linear Discriminant Analysis to create Fisherfaces which represent all the training data. One of the major differences between PCA and LDA is that PCA ignores classes. LDA treats the data in a way such that same classes are clustered tightly together, while different classes are as far away as possible from each other (Wagner, 2012).

Philipp Wagner supplied an implementation to Fisherfaces that proved very helpful in the implementation of this algorithm (Wagner, 2012). This algorithm attempts to create a projection where the within class scatter is minimized and the between class scatter is maximized (Belhumeur, 1997). The within class covariance matrix is given by equation (4), where $S_{\mathrm{w}}$ is the within class scatter matrix. $\mathrm{C}$ refers the total number of classes in the dataset. The $\mathrm{X}_{i j}$ represents the sample in the $i$ th position within class $j . \mu_{\mathrm{j}}$ represents the mean of class $j$. Before the cross product can be taken, the matrix must first be transposed, T. In equation (5), the between class scatter matrix $S_{b}$ is created. Here the mean of each class $\mu_{\mathrm{j}}$ is being subtracted by the total mean $\mu$.

$$
\begin{aligned}
& S_{w}=\sum_{j=1}^{C} \sum_{i=1}^{n_{j}}\left(X_{i j}-\mu_{j}\right)\left(X_{i j}-\mu_{j}\right)^{T} \\
& S_{b}=\sum_{j=1}^{C} N_{i}\left(\mu_{j}-\mu\right)\left(\mu_{j}-\mu\right)^{T}
\end{aligned}
$$

There is an issue where the within class scatter can become singular. This is because the number of images in the training set are usually much smaller than the number of pixels in each image (Belhumeur, 1997). The solution to this issue is to first use PCA on the data to reduce the dimensionality, then LDA can be used. The eigenvectors are extracted from the two scatters previously calculated. The NumPy library provides a useful method that will extract the eigenvectors from the covariance matrix. This method is numpy.linalg.eig(). To obtain the final 
projection, the eigenvectors obtained from PCA are multiplied by the eigenvectors obtained from the LDA process. Much like the Eigenfaces algorithm, each image can be decompiled into a linear combination of Fisherfaces (Belhumeur, 1997). Weights are calculated for all the faces in the training set. Once an image is input, the algorithm calculates the weights for each Fisherface. These weights are then compared to the weights in the training set. The face in the training set that has the closest distance to the input image is then chosen as the match. Figure 4 shows the procedure of this algorithm.

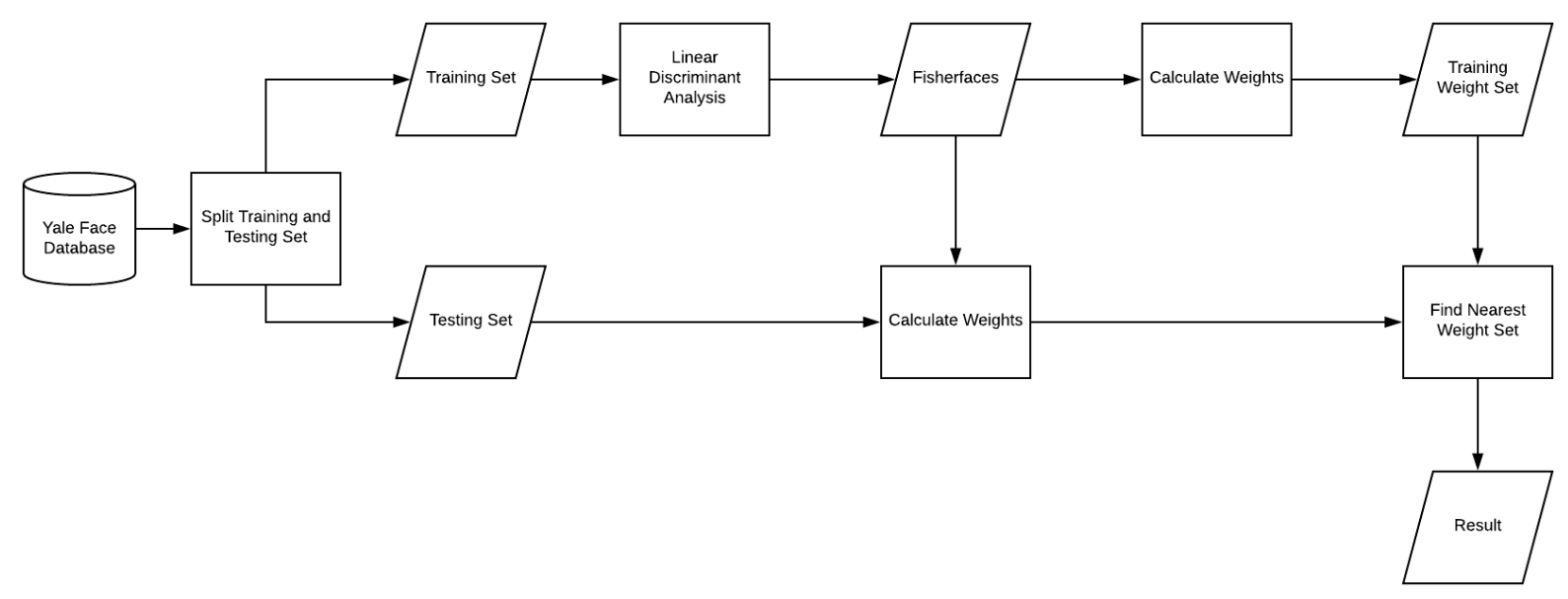

Figure 4: Fisherfaces Flow Chart 


\subsubsection{Local Binary Patterns Histograms Algorithm}

The Local Binary Patterns Histograms (LBPH) algorithm is a very simple yet powerful tool in face recognition. The LBPH algorithm takes the local binary patterns within the image, transforms these into histograms, then compares each histogram to recognize the face (Ahonen, 2006). There are two important values to keep in mind during this process: neighbors and block size. The local binary pattern of a single pixel is based on the pixels in its immediate neighborhood (Ahonen, 2006). For the purposes of explanation, the center pixel will be called the origin, and the outer pixels will be called the neighbors. In the case of this thesis, the number of neighbors will be set to 8 . Each image will be split up into blocks, these blocks will be the histograms, so the block size is set to $8 \times 8$. Ensuring that the block size is square will make things simple. The blocks at the edge of the image may be a little smaller assuming the image doesn't divide evenly into 8 .

The training phase of this algorithm involves creating another image that holds all the local binary patterns from the original image. To obtain the LBP, the origin pixel must be compared to each of its neighbors. If the neighbor has a value greater than the value of the origin, then it is denoted as a 1. On the other hand, if the neighbor's value is less, then the value will be denoted as a 0 . At the end of this process, a set of 8 numbers will be obtained. This 8 -digit binary number serves as the LBP for that specific pixel. This procedure can be seen in Figure 5. 


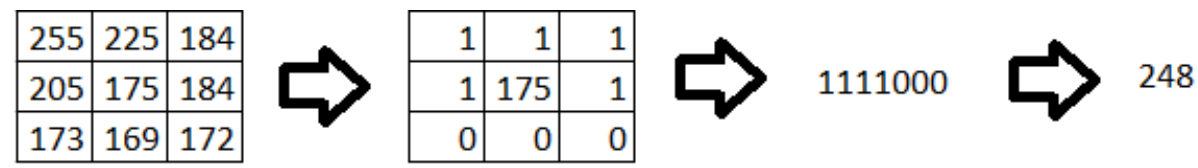

Figure 5: LBP process

The LBP are stored in the LBP image as a decimal number. This process is completed for every pixel in the image. An LBP image is created through this process. Figure 6 shows the initial face side by side with the new LBP face. Once the LBP image is completely calculated, it is then split up into blocks. Each of these blocks are flattened so that they can represent a histogram. Each histogram in the image is then connected giving a resulting cumulative histogram. This process of creating an LBP image and transforming it into a histogram is completed for each image in the dataset. This training process can be very time consuming.
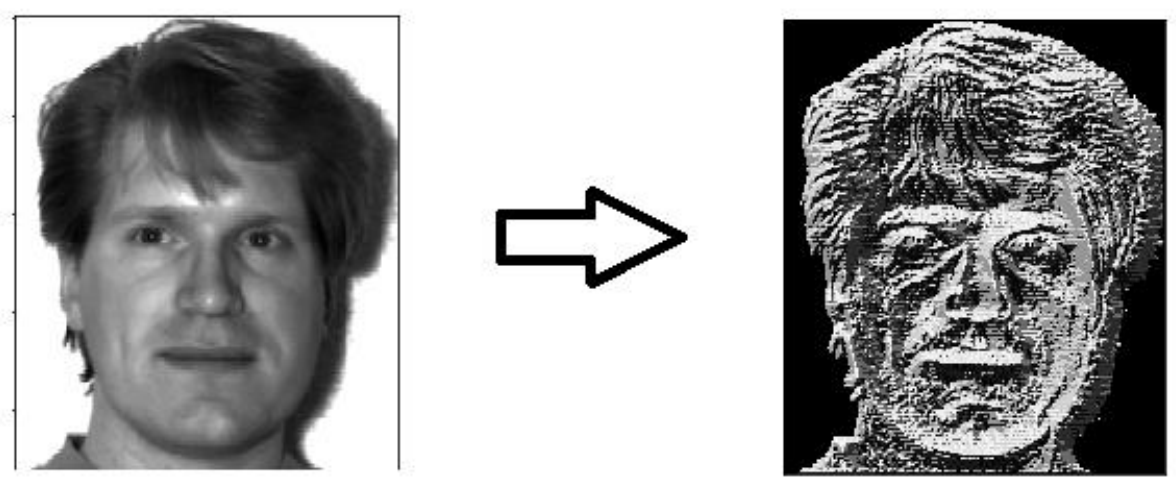

Figure 6: LBP transformation (Yale Face Database, n.d.)

Face recognition in the LBPH classifier follows the same steps as the training phase. Using the steps previously discussed, the input image is transformed into an LBP image. The LBP image is then translated into a histogram. The next step is to find the histogram that most closely resembles the histogram of the input image. The approach used to complete this task is to 
find the Euclidean distance. The histogram with the shortest distance is considered to be the class of the input image. Figure 7 shows the inner process of this algorithm.

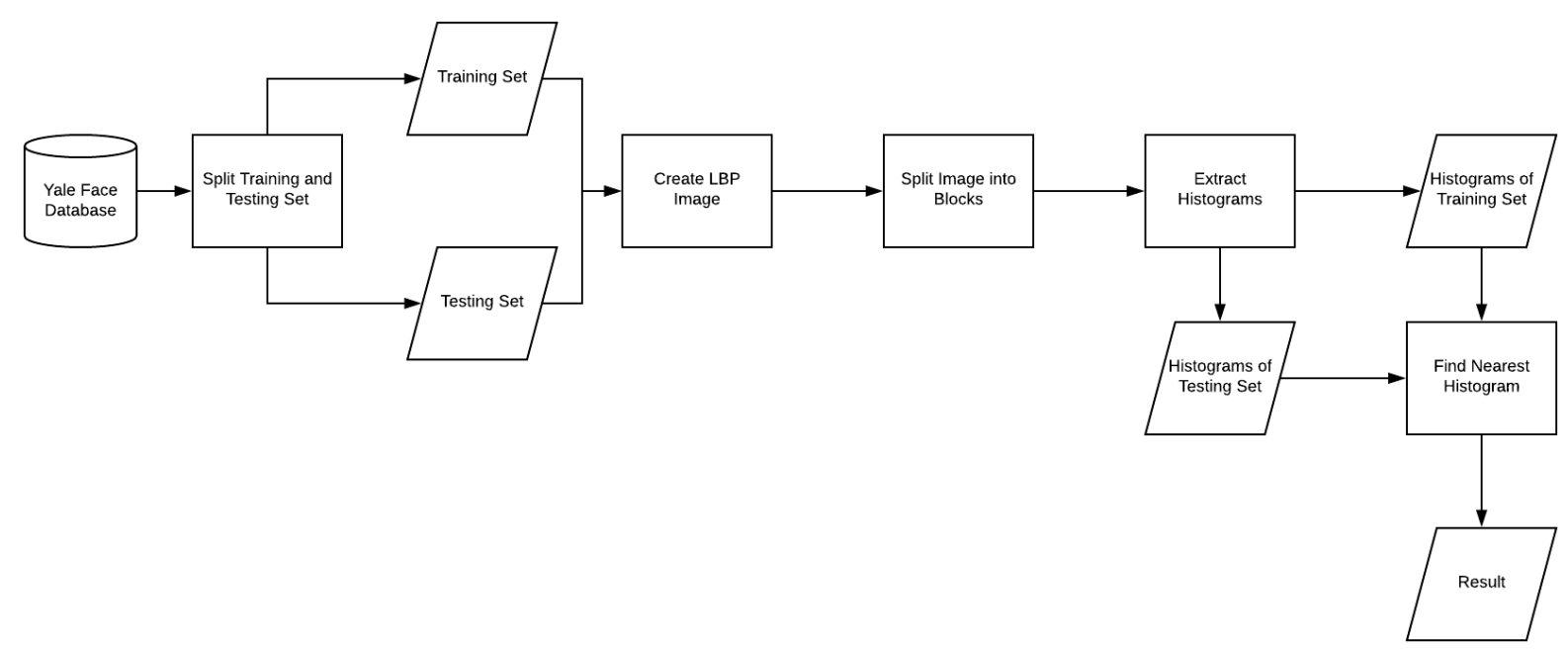

Figure 7: Local Binary Patterns Histograms Flow Chart

\subsubsection{EFL Hybrid Algorithm}

The three previously described algorithms are more than capable of handling face recognition requests on their own; however, they aren't perfect. They all work towards similar goals, but sometimes provide different experimental results. The last objective of this thesis is to design and implement a hybrid algorithm that combines the three previously discussed algorithms. The goal of this algorithm is to improve upon the accuracy of the three previous classifiers by combination. When developing new algorithms reinventing the wheel can be troublesome and may not even yield better experimental results. An easier solution is to simply combine algorithms. This new algorithm will be referred to as the EFL Hybrid algorithm, based on the first letters of the algorithms it contains. 
The EFL Hybrid algorithm combines the three classifiers: Eigenfaces, Fisherfaces, and Local Binary Patterns Histograms. Each classifier votes on a result based on the given input. The class with the greatest number of votes is then given as the combined result. An issue can arise if all three of the classifiers provide a different class, resulting in a three-way tie. A method in alleviating this issue is to rank the classifiers in a such a way that if there is no majority, the highest ranked algorithm's vote wins. An overview of the EFL algorithm can be seen in Figure 8.

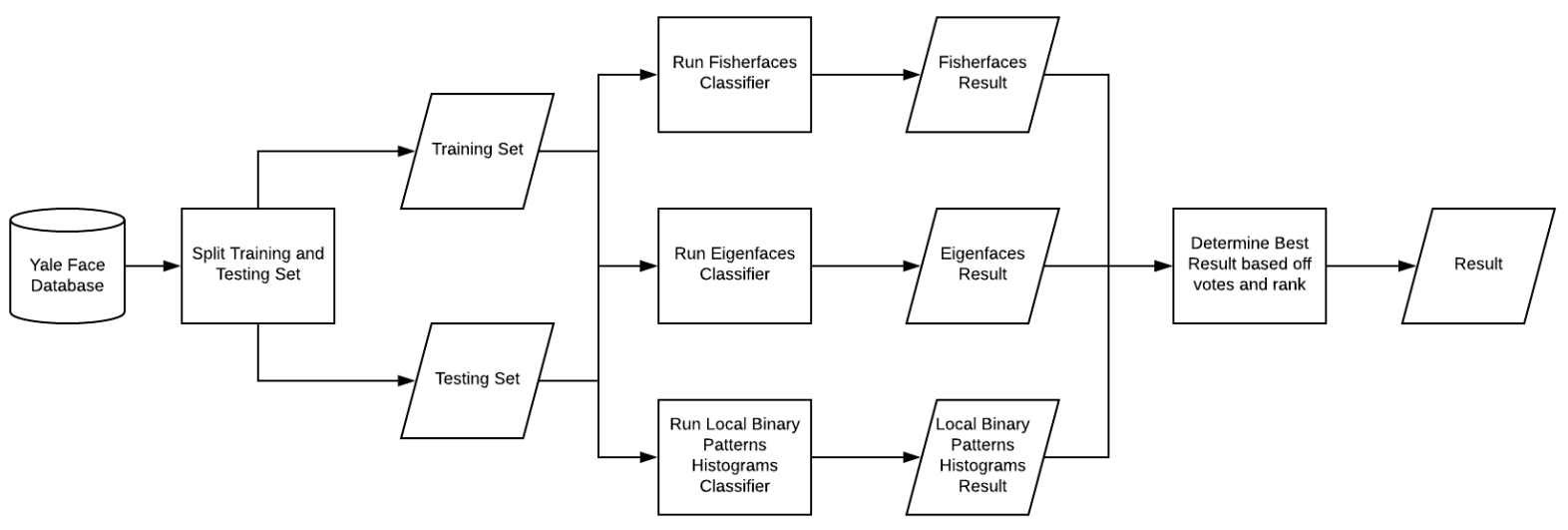

Figure 8: EFL Hybrid Flow Chart 


\section{CHAPTER IV: EXPERIMENTAL RESULTS}

\subsection{Database for Face Recognition}

One of the objectives of this thesis is to test and analyze the experimental results from the implemented face recognition classifiers. To achieve this objective, data needs to be collected. The facial recognition algorithm will need plenty of face data to have the best possible classification results. Fortunately, there are many face databases available on the internet for free. The database that will be used for this experiment is The Yale Face Database. This database contains 165 images with 15 different subjects, each subject having 11 images (Yale Face Database, n.d.). Figure 9 shows a sample set of these faces. This sample set is all for a single person, showing the different kinds of variation that will be tested in the coming classifiers. These variations include: facial expressions, background shadows, and glasses. This dataset will provide plenty of examples to train the algorithm.

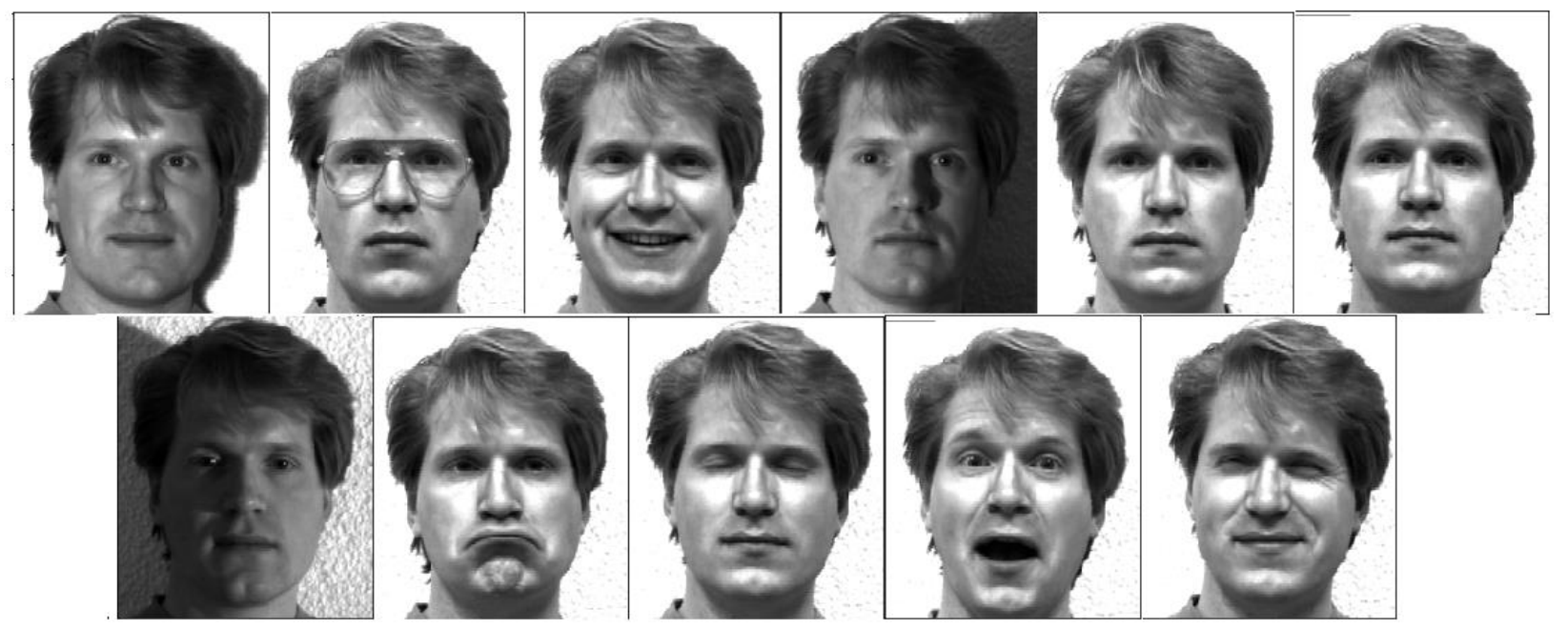

Figure 9: Yale Database Sample Data (Yale Face Database, n.d.) 


\subsection{Eigenfaces Experimental Results}

The very first experiment of this thesis is the testing of the Eigenfaces algorithm. Using the methodology discussed in the previous section, an implementation of the algorithm was created. Principal Component Analysis was applied on the dataset provided by Yale (Yale Face Database, n.d.) to obtain the Eigenfaces. Figure 10 shows the Eigenfaces that were extracted. These faces contain all the principal components of the training set. Any image in the training set can be reconstructed by taking a combination of these Eigenfaces (Turk, 1991).

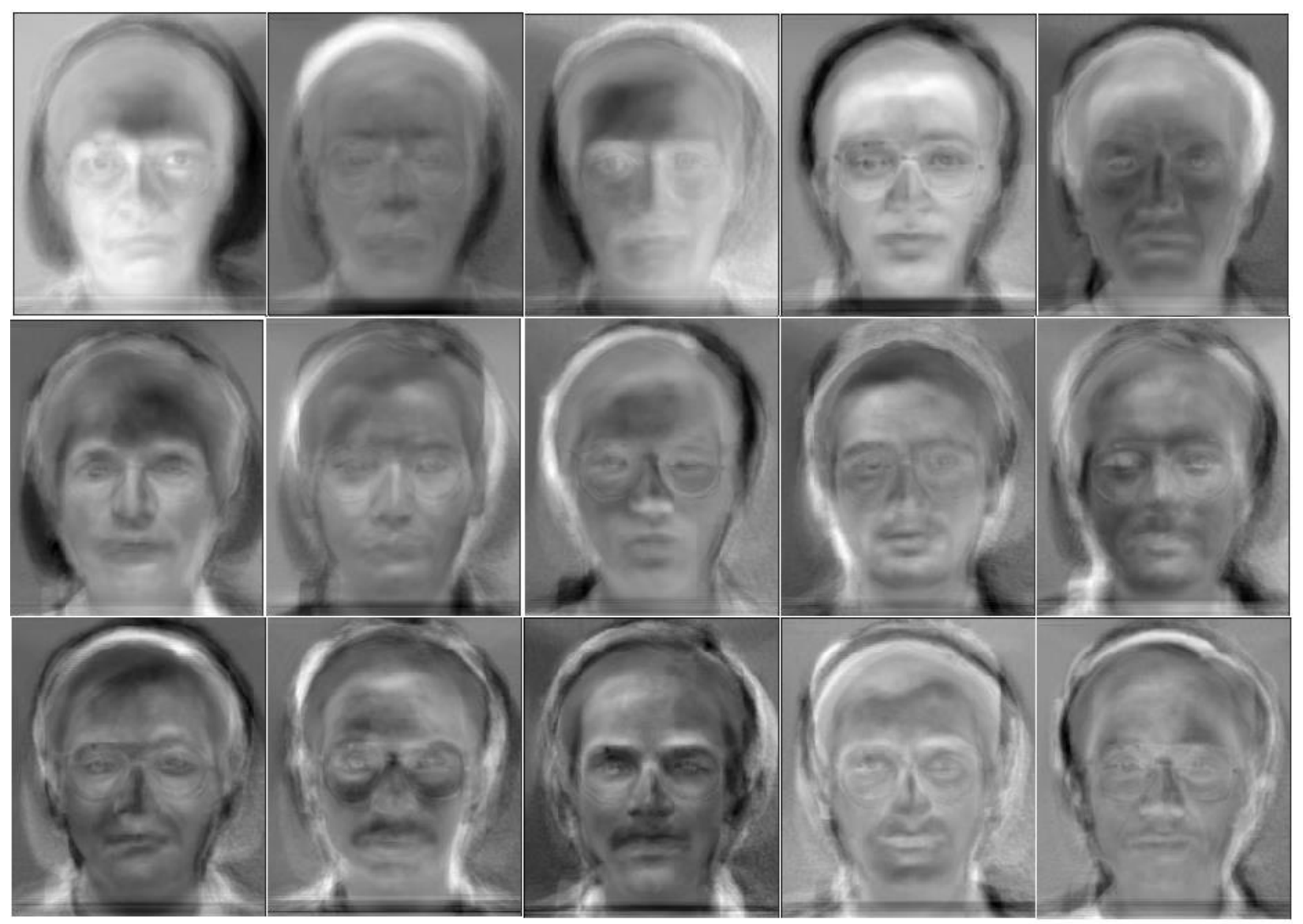

Figure 10: Eigenfaces (Yale Face Database, n.d.) 
As can be seen in Table 1, employing this algorithm on the Yale Face Database results in an accuracy of $83 \%$. Depending on how strict of a system, these experimental results may not be acceptable. Consider a case where this face recognition technology is used as a form of access control. False negatives would be just an annoyance for the user; however, a false positive can lead to a user having access to systems he has no authorization for. Using this line of reasoning, this algorithm doesn't provide acceptable results. Both the training phase and the testing phase of this algorithm is very fast.

Table 1: Algorithm Accuracy on Yale Database

\begin{tabular}{|c|c|c|}
\hline Eigenfaces & Fisherfaces & Local Binary Patterns Histograms \\
\hline 0.83 & 0.92 & 0.92 \\
\hline
\end{tabular}

Figure 11 shows the confusion matrix on this algorithm, allowing us to see where the errors occurred. Figure 12 provides more detail for which faces were unable to be classified properly. All these images that caused errors, save one, had some sort of shadow effect going on in the background. It did perform well on faces that had different facial expression. It can be concluded that this algorithm is too sensitive to the effects of the surroundings and should only be used when a consistent background can be used. 


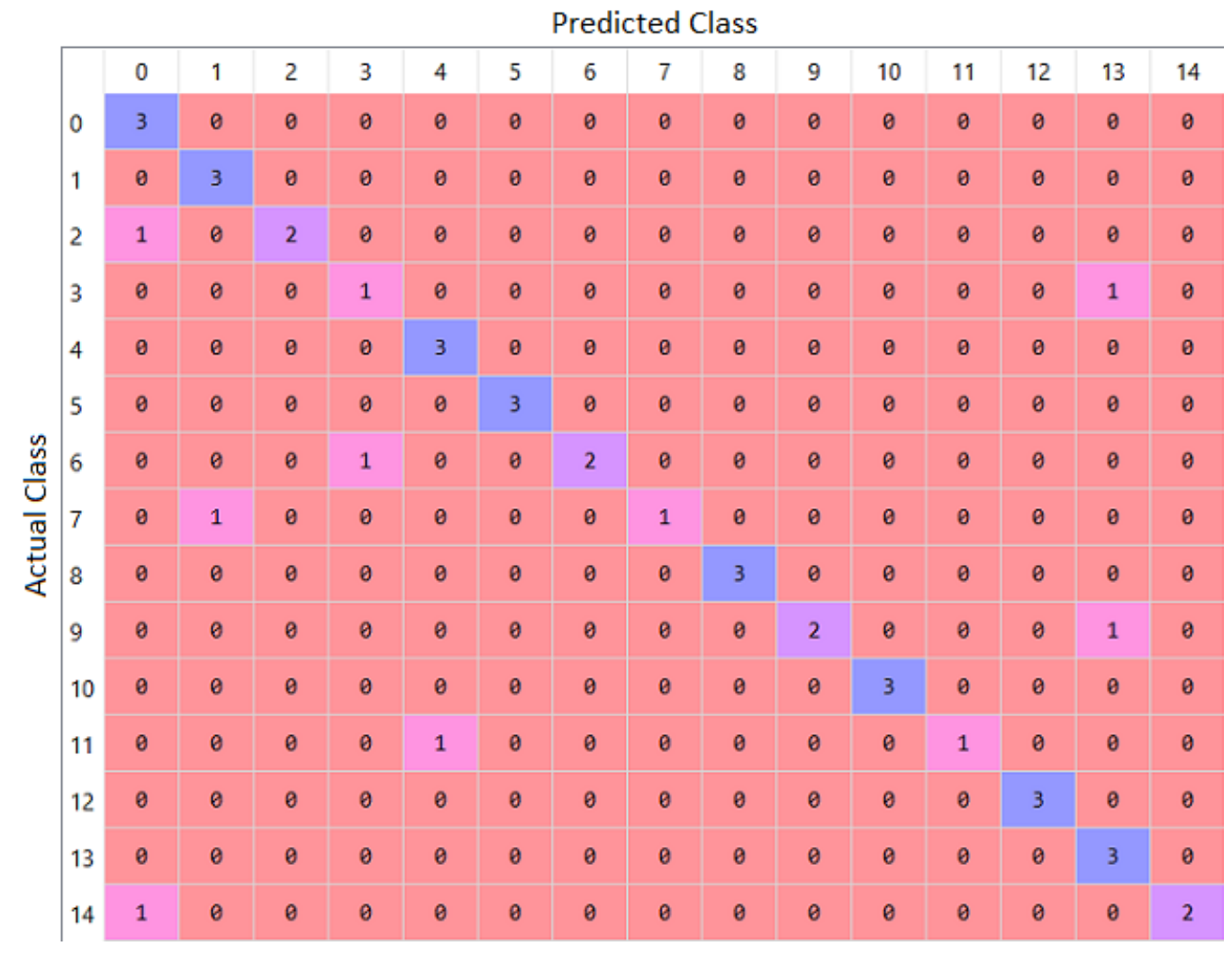

Figure 11: Eigenfaces Confusion Matrix 


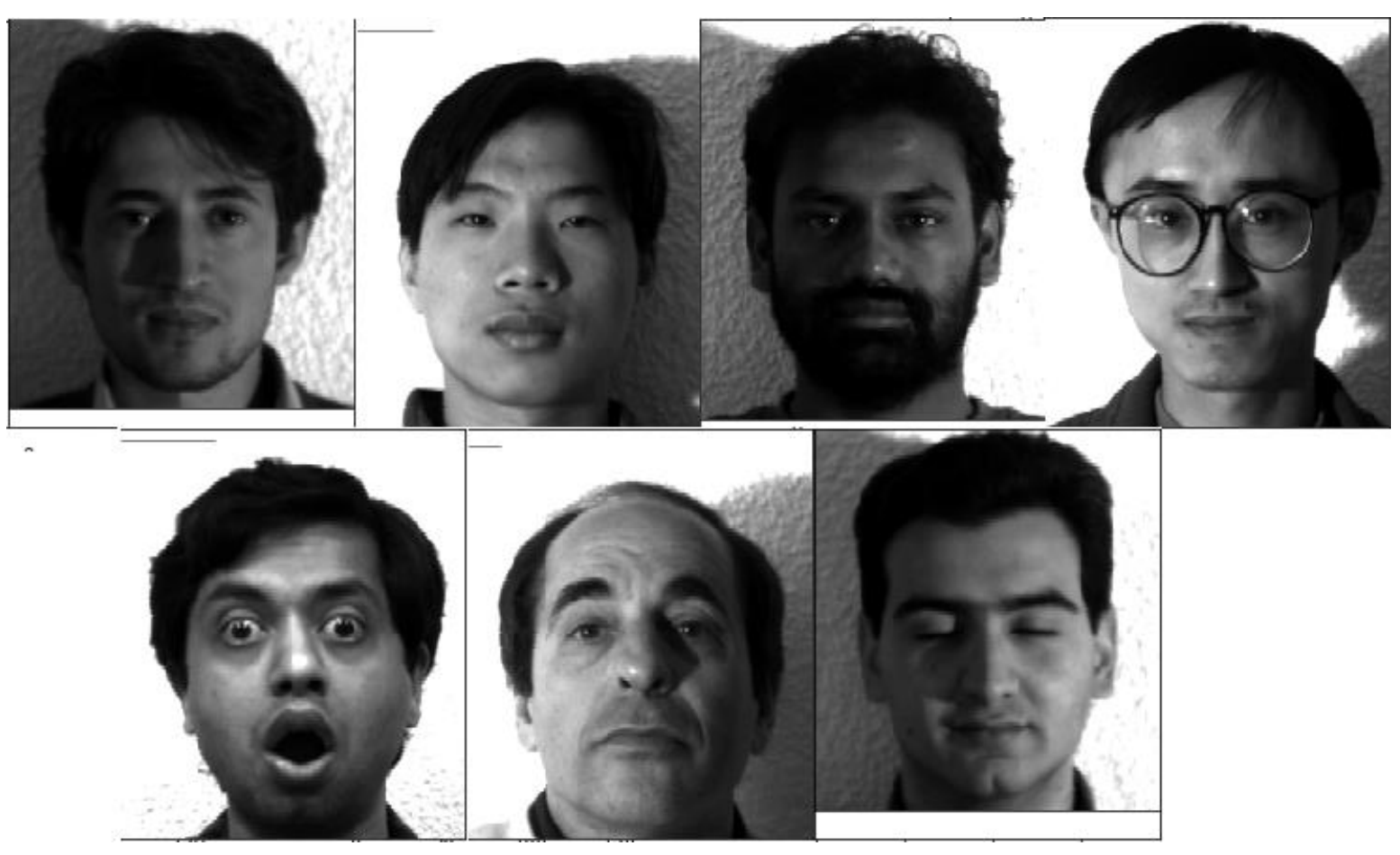

Figure 12: Eigenfaces Errors (Yale Face Database, n.d.)

\subsection{Fisherfaces Experimental Results}

The next experiment to be completed was the testing of the Fisherfaces algorithm. Philip Wagner provided a useful implementation to this algorithm that the classifier created for the purposed of this experiment was heavily based off (Wagner, 2012). This classifier is much more complex than the Eigenfaces, so better experimental results are expected. After applying Linear Discriminant Analysis on the Yale database (Yale Face Database, n.d.), the Fisherfaces can then be extracted. Figure 13 shows the Fisherfaces that were obtained. 


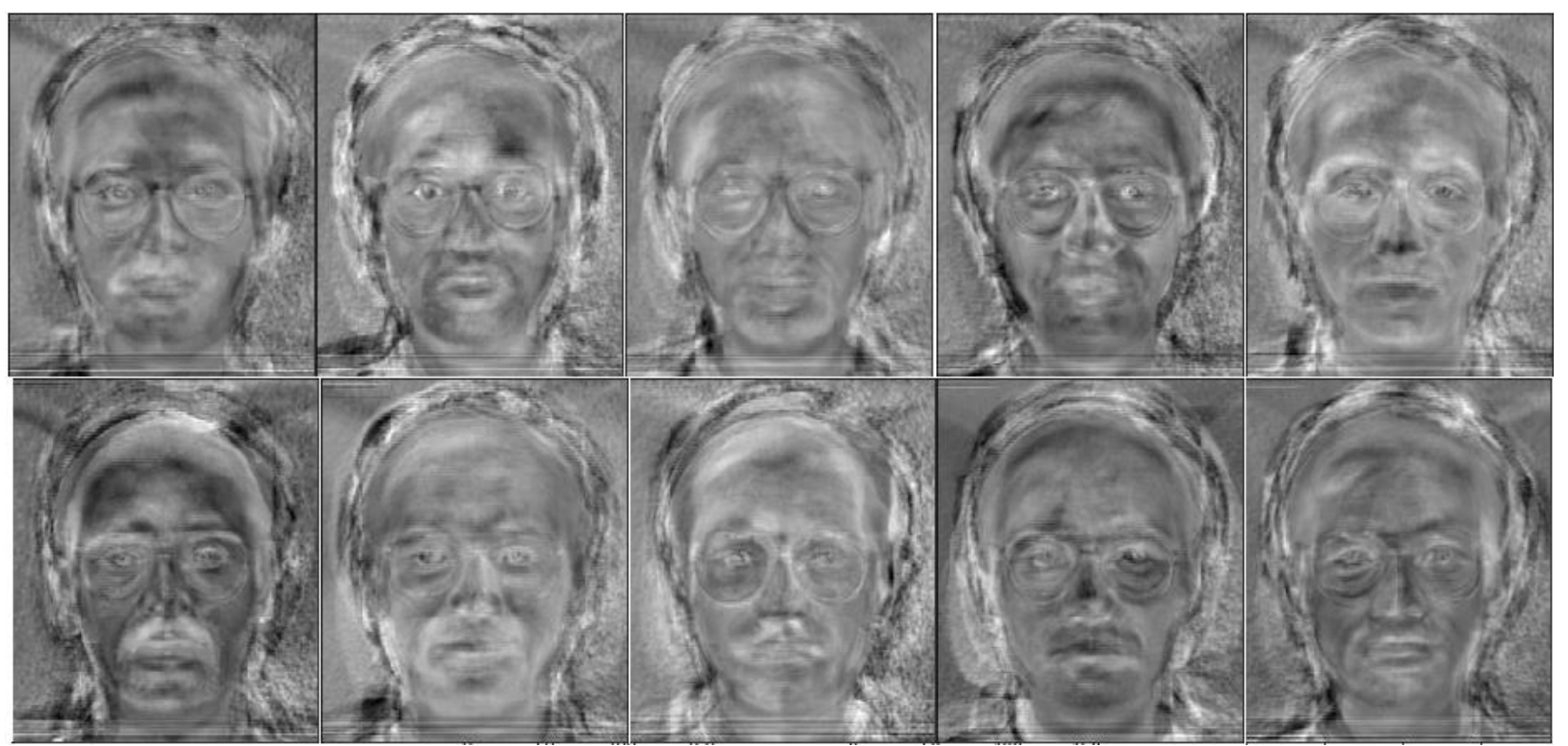

Figure 13: Fisherfaces (Yale Face Database, n.d.)

After employing these Fisherfaces an accuracy of $92 \%$ was obtained, as can be seen in table 1. Figure 14 shows the confusion matrix obtained from the Fisherfaces algorithm. Out of the 43 samples in the testing set there were only 3 errors. This is a major improvement over the Eigenfaces result. Figure 15 shows the errors that resulted from the use of this classifier. Two faces make a reappearance from the Eigenfaces error list. It appears that the background shadows can still cause issues within this face recognition classifier; however, it doesn't have as big hold as it did in the previous algorithm. Interestingly, two of the faces on the error list have a nonneutral facial expression. A combination of the shadow background and facial expressions may also sometimes cause a problem; however, most were classified properly. This classifier also runs very fast on the Yale Face Database image set. It runs just as fast as the Eigenfaces algorithm, so that leave very little reason to use that classifier. 
Predicted Class

\begin{tabular}{|c|c|c|c|c|c|c|c|c|c|c|c|c|c|c|c|}
\hline & 0 & 1 & 2 & 3 & 4 & 5 & 6 & 7 & 8 & 9 & 10 & 11 & 12 & 13 & 14 \\
\hline 0 & 3 & 0 & 0 & $\theta$ & 0 & 0 & 0 & 0 & 0 & 0 & 0 & 0 & 0 & 0 & 0 \\
\hline 1 & 0 & 3 & 0 & 0 & 0 & $\theta$ & $\theta$ & 0 & 0 & 0 & 0 & $\theta$ & $\theta$ & $\theta$ & 0 \\
\hline 2 & 0 & 0 & 2 & 0 & $\theta$ & $\theta$ & 0 & 0 & $\theta$ & 0 & 0 & 0 & 0 & 1 & 0 \\
\hline 3 & 0 & 0 & 0 & 2 & 0 & $\theta$ & 0 & 0 & 0 & 0 & 0 & 0 & 0 & 0 & 0 \\
\hline 4 & 0 & 0 & 0 & 0 & 3 & 0 & 0 & 0 & 0 & 0 & 0 & $\theta$ & $\theta$ & 0 & 0 \\
\hline 5 & 0 & 0 & 0 & $\theta$ & 0 & 3 & $\theta$ & 0 & 0 & 0 & 0 & $\theta$ & 0 & 0 & 0 \\
\hline 6 & 0 & $\theta$ & 0 & $\theta$ & 0 & $\theta$ & 2 & 0 & 0 & 0 & 0 & 0 & 1 & 0 & 0 \\
\hline 7 & 0 & 0 & 0 & 0 & 0 & 0 & 0 & 2 & 0 & 0 & 0 & 0 & 0 & 0 & 0 \\
\hline 8 & 0 & 0 & 0 & 0 & 0 & $\theta$ & 0 & 0 & 3 & 0 & 0 & $\theta$ & $\theta$ & $\theta$ & 0 \\
\hline 9 & 0 & 0 & 0 & $\theta$ & $\theta$ & 0 & $\theta$ & 0 & 0 & 3 & 0 & 0 & 0 & 0 & 0 \\
\hline 10 & 0 & 0 & 0 & 0 & 0 & $\theta$ & 0 & 0 & 0 & 0 & 3 & 0 & 0 & 0 & 0 \\
\hline 11 & 0 & 0 & 0 & 0 & 0 & 0 & 0 & 0 & 0 & 0 & 0 & 2 & 0 & 0 & 0 \\
\hline 12 & 0 & 0 & 0 & $\theta$ & 0 & $\theta$ & 0 & 0 & 0 & 0 & 0 & $\theta$ & 3 & 0 & 0 \\
\hline 13 & 0 & 0 & 0 & $\theta$ & 0 & $\theta$ & 0 & 0 & 0 & 0 & 0 & $\theta$ & $\theta$ & 3 & 0 \\
\hline 14 & 0 & 0 & 0 & 0 & 0 & 0 & 0 & 0 & 0 & 0 & 0 & 0 & 1 & 0 & 2 \\
\hline
\end{tabular}

Figure 14: Fisherface Confusion Matrix
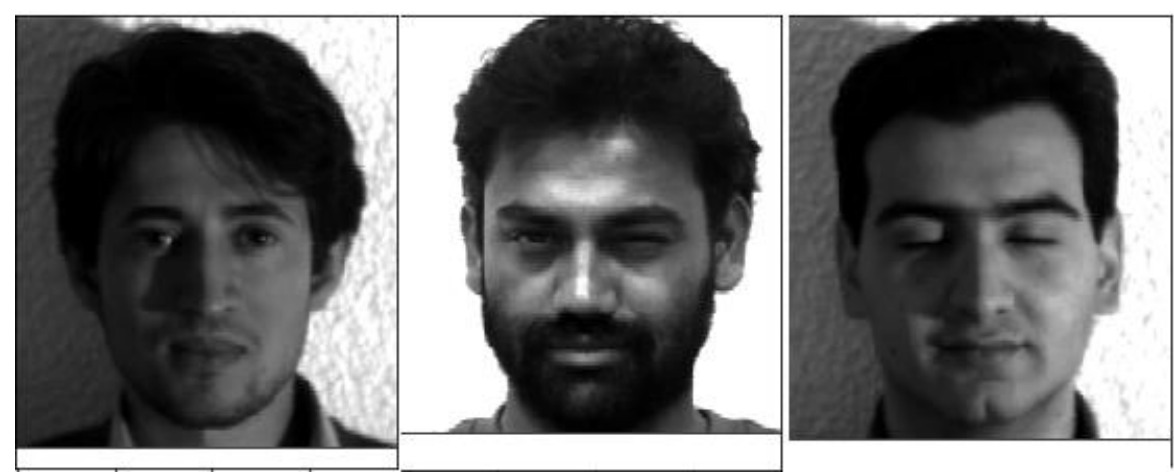

Figure 15: Fisherface Errors (Yale Face Database, n.d.) 


\subsection{Local Binary Patterns Histogram Experimental Results}

While the Fisherfaces result was very good, the next experiment will hopefully provide a better accuracy. The Local Binary Patterns Histogram is the next classifier that is implemented. The methodology described for this algorithm may seem a little bit complicated at first glance; however, implementing isn't overly complicated. In table 1 we can see that using this classifier on the data from the Yale Face Database provides a 92\% accuracy. This provides the same result as the Fisherfaces classifier. Figure 16 shows the confusion matrix obtained from this classifier. While it provided the same accuracy as the Fisherfaces algorithm, it has obtained errors on mostly different images. As can be seen from the LBPH errors in Figure 17, once again the shadows in the background are causing the occasional error. Interestingly the closed eyes image was misclassified by all three of the previous algorithms. During the training phase, this algorithm runs extremely slow. It takes more a minute to train this algorithm, compared the Fisherfaces which trains in about a second. Testing the algorithm on the Yale Database takes almost 5 minutes to complete. While taking a long time to run it provides about the same accuracy as the Fisherfaces algorithm. 
Predicted Class

\begin{tabular}{|c|c|c|c|c|c|c|c|c|c|c|c|c|c|c|c|}
\hline & 0 & 1 & 2 & 3 & 4 & 5 & 6 & 7 & 8 & 9 & 10 & 11 & 12 & 13 & 14 \\
\hline 0 & 3 & 0 & 0 & 0 & 0 & $\theta$ & 0 & 0 & $\theta$ & 0 & 0 & $\theta$ & 0 & $\theta$ & 0 \\
\hline 1 & 0 & 3 & 0 & 0 & 0 & $\theta$ & 0 & 0 & 0 & 0 & 0 & 0 & 0 & 0 & 0 \\
\hline 2 & $\theta$ & 0 & 3 & $\theta$ & 0 & $\theta$ & $\theta$ & 0 & $\theta$ & 0 & 0 & $\theta$ & 0 & $\theta$ & $\theta$ \\
\hline 3 & $\theta$ & 0 & 0 & 2 & 0 & $\theta$ & $\theta$ & 0 & 0 & 0 & 0 & $\theta$ & $\theta$ & $\theta$ & $\theta$ \\
\hline 4 & 0 & 0 & 0 & 0 & 3 & 0 & 0 & 0 & 0 & 0 & 0 & 0 & 0 & 0 & 0 \\
\hline 5 & 0 & 0 & 0 & 0 & 0 & 3 & $\theta$ & 0 & 0 & 0 & 0 & $\theta$ & 0 & $\theta$ & 0 \\
\hline 6 & $\theta$ & 0 & 0 & $\theta$ & 0 & 0 & 3 & $\theta$ & $\theta$ & $\theta$ & 0 & $\theta$ & $\theta$ & $\theta$ & 0 \\
\hline 7 & 0 & 0 & 0 & 0 & 0 & $\theta$ & 0 & 2 & 0 & 0 & 0 & $\theta$ & 0 & 0 & 0 \\
\hline 8 & 0 & 0 & 0 & $\theta$ & 0 & 0 & $\theta$ & 0 & 2 & 0 & 0 & $\theta$ & 0 & 1 & 0 \\
\hline 9 & $\theta$ & 0 & 0 & $\theta$ & $\theta$ & $\theta$ & $\theta$ & 0 & $\theta$ & 3 & 0 & $\theta$ & $\theta$ & $\theta$ & 0 \\
\hline 10 & 0 & 0 & 0 & $\theta$ & 0 & $\theta$ & $\theta$ & 0 & $\theta$ & 0 & 3 & $\theta$ & 0 & 0 & 0 \\
\hline 11 & 0 & 0 & 0 & 0 & 1 & $\theta$ & 0 & 0 & 0 & 0 & 0 & 1 & 0 & 0 & 0 \\
\hline 12 & 0 & 0 & 0 & $\theta$ & 0 & $\theta$ & $\theta$ & 0 & $\theta$ & 0 & 0 & $\theta$ & 3 & $\theta$ & 0 \\
\hline 13 & $\theta$ & 0 & 0 & $\theta$ & 0 & $\theta$ & $\theta$ & 0 & $\theta$ & 0 & 0 & $\theta$ & 0 & 3 & $\theta$ \\
\hline 14 & 0 & 0 & 0 & 0 & 0 & $\theta$ & 0 & 0 & 0 & 1 & 0 & 0 & 0 & 0 & 2 \\
\hline
\end{tabular}

Figure 16: LBPH Confusion Matrix

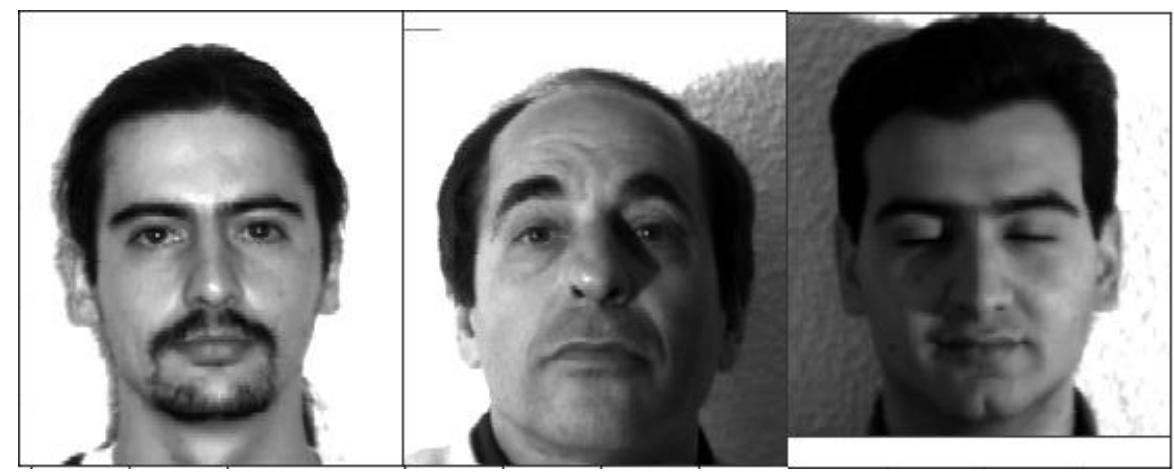

Figure 17: LBPH Errors (Yale Face Database, n.d.) 


\subsection{EFL Hybrid Algorithm Experimental Results}

Table 2: EFL Hybrid Algorithms Accuracy Based on Highest Rank

\begin{tabular}{|c|c|c|}
\hline Ranked Eigenfaces & Ranked Fisherfaces & Ranked Local Binary Patterns Histograms \\
\hline 0.92 & 0.92 & 0.95 \\
\hline
\end{tabular}

The final algorithm to be implemented is the EFL Hybrid classifier. As was stated previously, there are three methods for creating this algorithm. Because a voting system is being used, there is a chance that all three algorithms vote for a different class, which would lead to a three-way tie. Each algorithm is given a rank so that in the case of a tie, the highest ranked algorithm's vote would be declared the winner. Our last experiment will be an implementation of three possible algorithms with a different classifier as the highest ranked. The experimental results obtained from using the different variations of the EFL classifier on the Yale Face Database are displayed in table 2. The algorithm that ranked the Eigenfaces vote as the most important yielded an accuracy of $92 \%$. While this is a big improvement over the Eigenfaces' results, it still provides the same results as both the Fisherfaces and the LBPH classifiers. This algorithm wouldn't be worth using because it takes more processing time for similar results. The EFL algorithm which ranked the Fisherfaces vote as the highest provided the same accuracy of $92 \%$. For the same reasoning stated previously, this is obviously not a useful classifier. The final algorithm is the LBPH ranked classifier. This classifier provides an accuracy of 95\%. The EFL algorithm that ranks LBPH the highest provides the best accuracy. Figure 18 shows the confusion matrix for this algorithm. If compared with Figure 16, it looks very similar. Employing the Eigenfaces and Fisherfaces algorithms managed to cut out one of the errors that previously existed in the LBPH classifier. Figure 19 shows the comparison of the accuracies of 
each algorithm. The EFL algorithm provides an accuracy that is slightly greater than the accuracy all its components; however, this boost in accuracy comes at the expense of higher computational time.

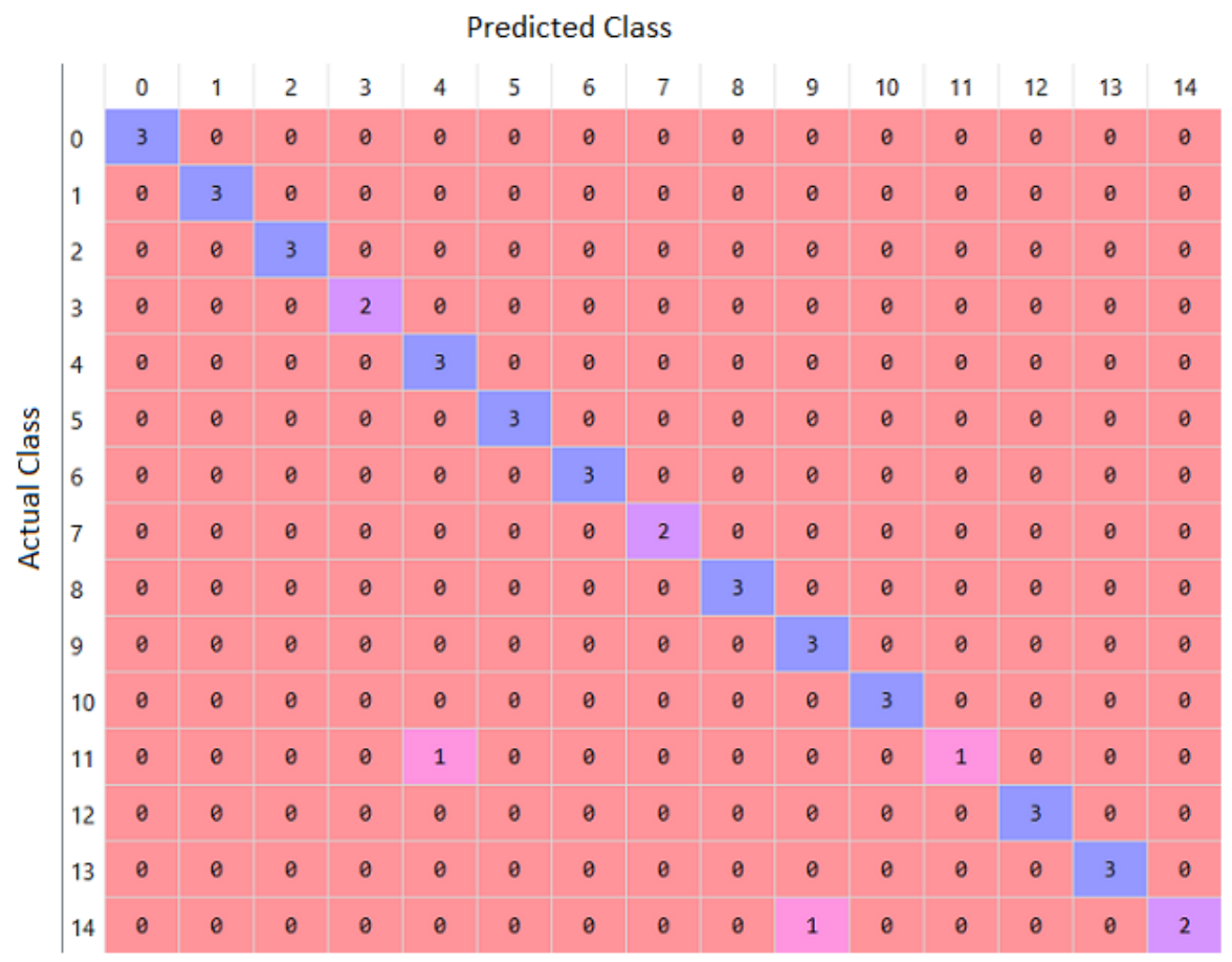

Figure 18: EFL Hybrid Algorithm using LBPH rank Confusion Matrix 


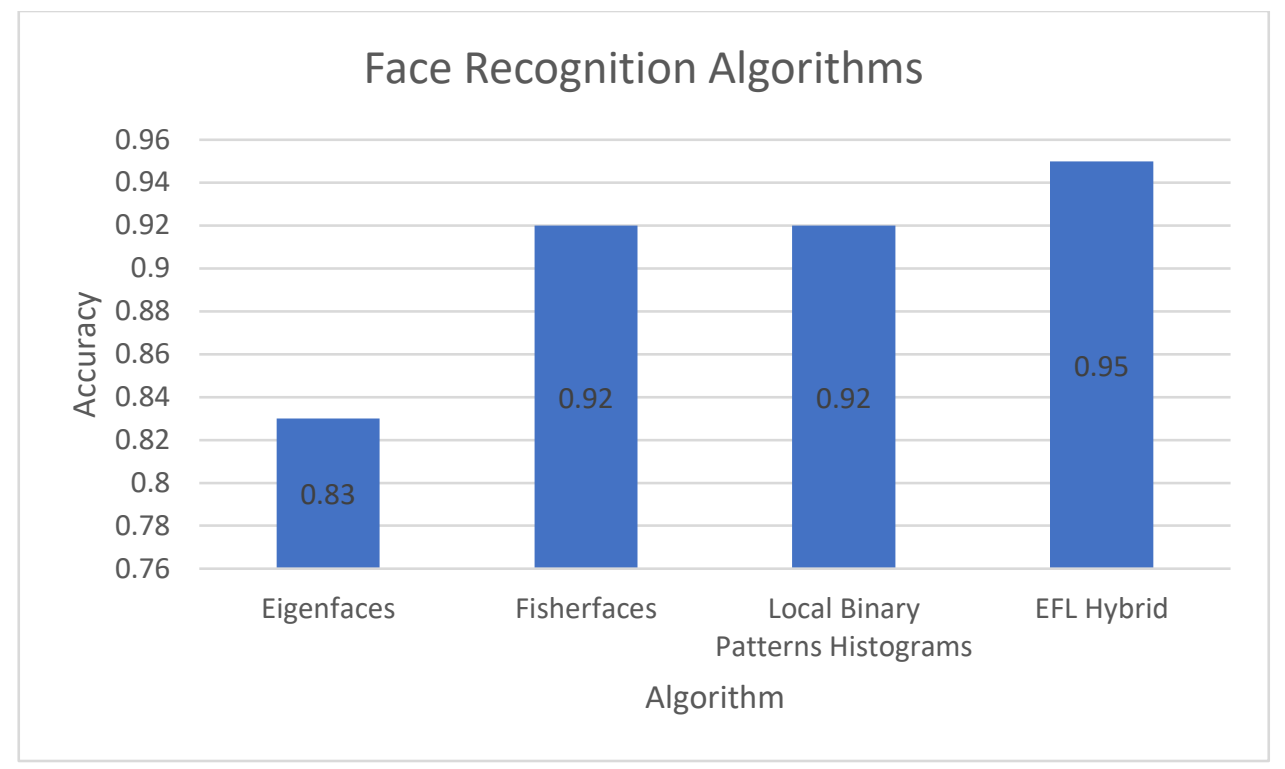

Figure 19: Comparison of Algorithms

Table 3 displays the run time for each algorithm. In all cases barring the Eigenfaces, the testing phase appears to take longer than the training phase. Both the Eigenfaces and the Fisherfaces algorithms run almost instantaneously, whereas the LBPH and the EFL Hybrid algorithms are much slower. The cost of obtaining a higher accuracy from the EFL Hybrid is the longer run times.

Table 3: Algorithm Speed (seconds)

\begin{tabular}{|c|c|c|c|c|}
\hline & Eigenfaces & Fisherfaces & Local Binary Patterns Histograms & EFL Hybrid \\
\hline $\begin{array}{c}\text { Training } \\
\text { Phase }\end{array}$ & 1.16 & 1.89 & 112.36 & 115.41 \\
\hline $\begin{array}{c}\text { Testing } \\
\text { Phase }\end{array}$ & 0.35 & 2.06 & 280.38 & 282.79 \\
\hline
\end{tabular}




\section{CHAPTER V: CONCLUSIONS}

\subsection{Summary}

There were three initial goals stated at the beginning of this thesis. Implementing three popular face recognition algorithms. Eigenfaces, Fisherfaces and Local Binary Patterns Histograms were the three algorithms that were implemented. Once these algorithms were completed, they were run on data obtained from the Yale Face Database (Yale Face Database, n.d.). The experimental results found that the Eigenface algorithm is significantly worse than both the Fisherface and the Local Binary Patterns Histograms. All the algorithms discussed were sensitive to shadows in the background. A consistent background may be necessary for optimal results. Both the Eigenfaces and the Fisherfaces algorithms run quite a bit faster than the Local Binary Patterns Histograms algorithm. Due to this fact, the Fisherfaces algorithm should be considered the best algorithm for this data set. The last goal was to combine these classifiers such that there is improvement over the originals. When ranking the different algorithms within the EFL hybrid classifier, both the Eigenfaces and Fisherfaces ranked algorithms didn't provide the experimental results that would warrant their usage. On the other hand, the LBPH ranked classifier provided a slight improvement over its' opponents. The EFL Hybrid classifier provides a small increase in accuracy for a bit longer loading time. As technology continues to grow and improve, the loading times will decrease but the accuracy will remain the same. Face recognition and machine learning are valuable tools in today's world, so having access to these algorithms can be very useful. 


\subsection{Future Work}

There are several ways we can improve with future work. Much of the errors that occurred utilizing these algorithms were caused by shadows in the background of the image. If the images are properly cropped such that the backgrounds become irrelevant, this may lead to better experimental results. In this thesis, only three algorithms were combined. Adding even more algorithms can be an option. The issue with combining more algorithms is the fact that it would increase the amount of processing time required. This may become more feasible as computer technology continues to be improved. An interesting concept would be comparing how much increased accuracy can be obtained at the expense of processing time. This thesis used a method of combining algorithms by taking the majority result from the classifiers. In future work, a algorithm can be created that takes the strengths of each of the separate components. Testing more face databases can also be explored in the future. 


\section{REFERENCES}

Ahonen, T., Hadid, A., \& Pietikäinen, M. (2006). Face Description with Local Binary Patterns: Application to Face Recognition. IEEE Transactions on Pattern Analysis and Machine Intelligence, 28, 2037-2041.

Anaconda. (2018, November 10). What is Anaconda? Retrieved from https://www.anaconda.com/what-is-anaconda/

Anggo, M., \& Arapu, L. (2018). Face Recognition Using Fisherface Method. Journal of Physics: Conference Series, 1028, 012119. doi:10.1088/1742-6596/1028/1/012119

Bicego, M., Lagorio, A., Grosso, E., \& Tistarelli, M. (2006). On the Use of SIFT Features for Face Authentication. 2006 Conference on Computer Vision and Pattern Recognition Workshop (CVPRW'06), 35-35.

Bradski, G., \& Kaehler, A. (2015). Learning OpenCV. OReilly Media.

Belhumeur, P.N., Hespanha, J.P., \& Kriegman, D.J. (1997). Eigenfaces vs. Fisherfaces: Recognition Using Class Specific Linear Projection. IEEE Trans. Pattern Anal. Mach. Intell., 19, 711-720.

Chan, T. F. (2018, March 16). 7-Eleven is bringing facial-recognition technology pioneered in China to its 11,000 stores in Thailand. Retrieved from https://www.businessinsider.com/7eleven-facial-recognition-technology-introduced-in-thailand-2018-3

Coffin, J. S., \& Ingram, D. (1999). U.S. Patent No. 5991429. Washington, DC: U.S. Patent and Trademark Office. 
D, Ambika \& B. Arathy. B, Arathy \& Ramalingam, Srinivasa Perumal. (2012). Comparison of PCA and MPCA with Different Databases for Face Recognition. International Journal of Computer Applications. 43. 30-34. 10.5120/6198-8730.

Du, G., Su, F., \& Cai, A. (2009). Face recognition using SURF features.

Fisher, R. A. (1936). The Use Of Multiple Measurements In Taxonomic Problems. Annals of Eugenics,7(2), 179-188. doi:10.1111/j.1469-1809.1936.tb02137.x

Hunter, J., \& Dale, D. (2007, May 27). The Matplotlib User's Guide. Retrieved from https://social.stoa.usp.br/articles/0015/3893/matplotlib.pdf

Jafri, R., Ali, S.A., \& Arabnia, H.R. (2013). Face Recognition for the Visually Impaired.

Jha, A. (2018). Class Room Attendance System Using Facial Recognition System. The International Journal of Mathematics, Science, Technology and Management,2(3).

Kantardzic, M. (2011). Data mining: concepts, models, methods and algorithms. Oxford: WileyBlackwell.

Kruszka, P., Addissie, Y. A., McGinn, D. E., Porras, A. R., Biggs, E., Share, M., Crowley, T. B., Chung, B. H., Mok, G. T., Mak, C. C., Muthukumarasamy, P., Thong, M. K., Sirisena, N. D., Dissanayake, V. H., Paththinige, C. S., Prabodha, L. B., Mishra, R., Shotelersuk, V., Ekure, E. N., Sokunbi, O. J., Kalu, N., Ferreira, C. R., Duncan, J. M., Patil, S. J., Jones, K. L., Kaplan, J. D., Abdul-Rahman, O. A., Uwineza, A., Mutesa, L., Moresco, A., Obregon, M. G., RichieriCosta, A., Gil-da-Silva-Lopes, V. L., Adeyemo, A. A., Summar, M., Zackai, E. H., McDonaldMcGinn, D. M., Linguraru, M. G., ... Muenke, M. (2017). 22q11.2 deletion syndrome in diverse populations. American journal of medical genetics. Part A, 173(4), 879-888. 
Lee, J. (2017, May 19). Australian passport authority developing facial recognition system.

Retrieved from https://www.biometricupdate.com/201705/australian-passport-authoritydeveloping-facial-recognition-system

Rahim, A., Md, Hossain, N., Md, Wahid, T., \& Azam, S., Md. (2013). Face Recognition using Local Binary Patterns (LBP). Global Journal of Computer Science and Technology Graphics \& Vision, 13(4).

Rahman, M.U. (2012). A comparative study on face recognition techniques and neural network. CoRR, abs/1210.1916.

Schroeder, C. C. (1998). U.S. Patent No. 5787186. Washington, DC: U.S. Patent and Trademark Office.

Turk, M., \& Pentland, A. (1991). Eigenfaces for Recognition. Journal of Cognitive Neuroscience,3(1), 71-86. doi:10.1162/jocn.1991.3.1.71

Wagner, P. (2012, July 18). Face Recognition with Python. Retrieved from https://bytefish.de/pdf/facerec_python.pdf

Walt, S. V., Colbert, S. C., \& Varoquaux, G. (2011). The NumPy Array: A Structure for Efficient Numerical Computation. Computing in Science \& Engineering,13(2), 22-30. doi:10.1109/mcse.2011.37

Wiskott, L., Fellous, J., Krüger, N., \& Malsburg, C. V. (1997). Face recognition by elastic bunch graph matching. Computer Analysis of Images and Patterns Lecture Notes in Computer Science,456-463. doi:10.1007/3-540-63460-6_150 
Wiskott, L., Würtz, R. P., \& Westphal, G. (2014). Elastic Bunch Graph Matching. Retrieved from http://www.scholarpedia.org/article/Elastic_Bunch_Graph_Matching

Wold, S., Esbensen, K., \& Geladi, P. (1987). Principal Component Analysis. Chemometrics and Intelligent Laboratory Systems.

Yale Face Database. (n.d.). Retrieved from http://vision.ucsd.edu/content/yale-face-database

Zhao, W., Krishnaswamy, A., Chellappa, R., Swets, D. L., \& Weng, J. (1998). Discriminant Analysis of Principal Components for Face Recognition. Face Recognition,73-85. doi:10.1007/978-3-642-72201-1_4

Zhang, W., Shan, S., Gao, W., Chen, X., \& Zhang, H. (2005). Local Gabor binary pattern histogram sequence (LGBPHS): a novel non-statistical model for face representation and recognition. Tenth IEEE International Conference on Computer Vision (ICCV'05) Volume 1, 1, 786-791 Vol. 1. 


\section{APPENDIX}

\section{Appendix A: Face Recognition Parent Class (Part 1)}

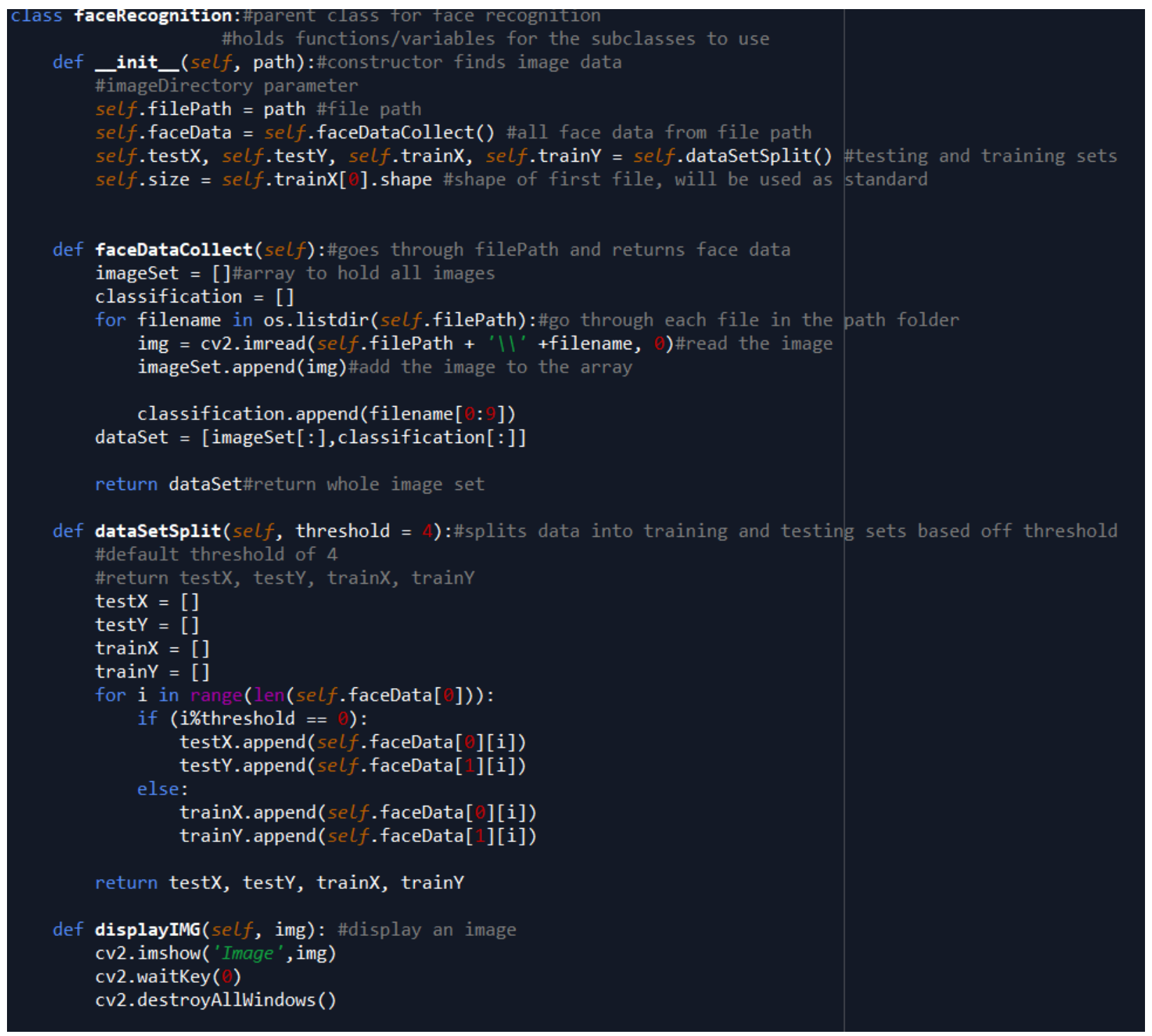




\section{Appendix B: Face Recognition Parent Class (Part 2)}

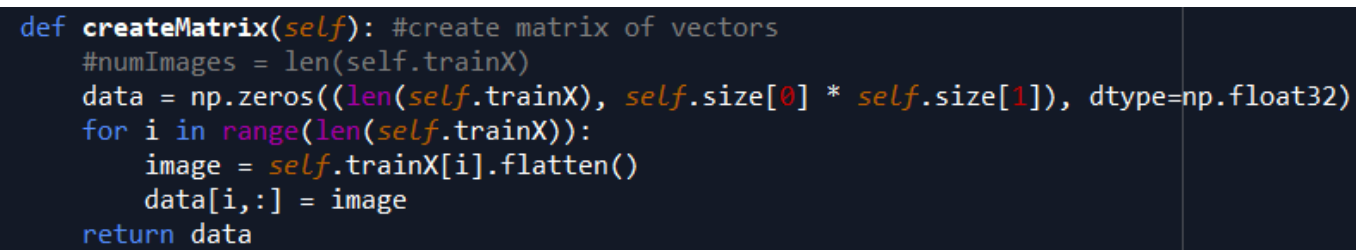




\section{Appendix C: Eigenfaces (Part 1)}

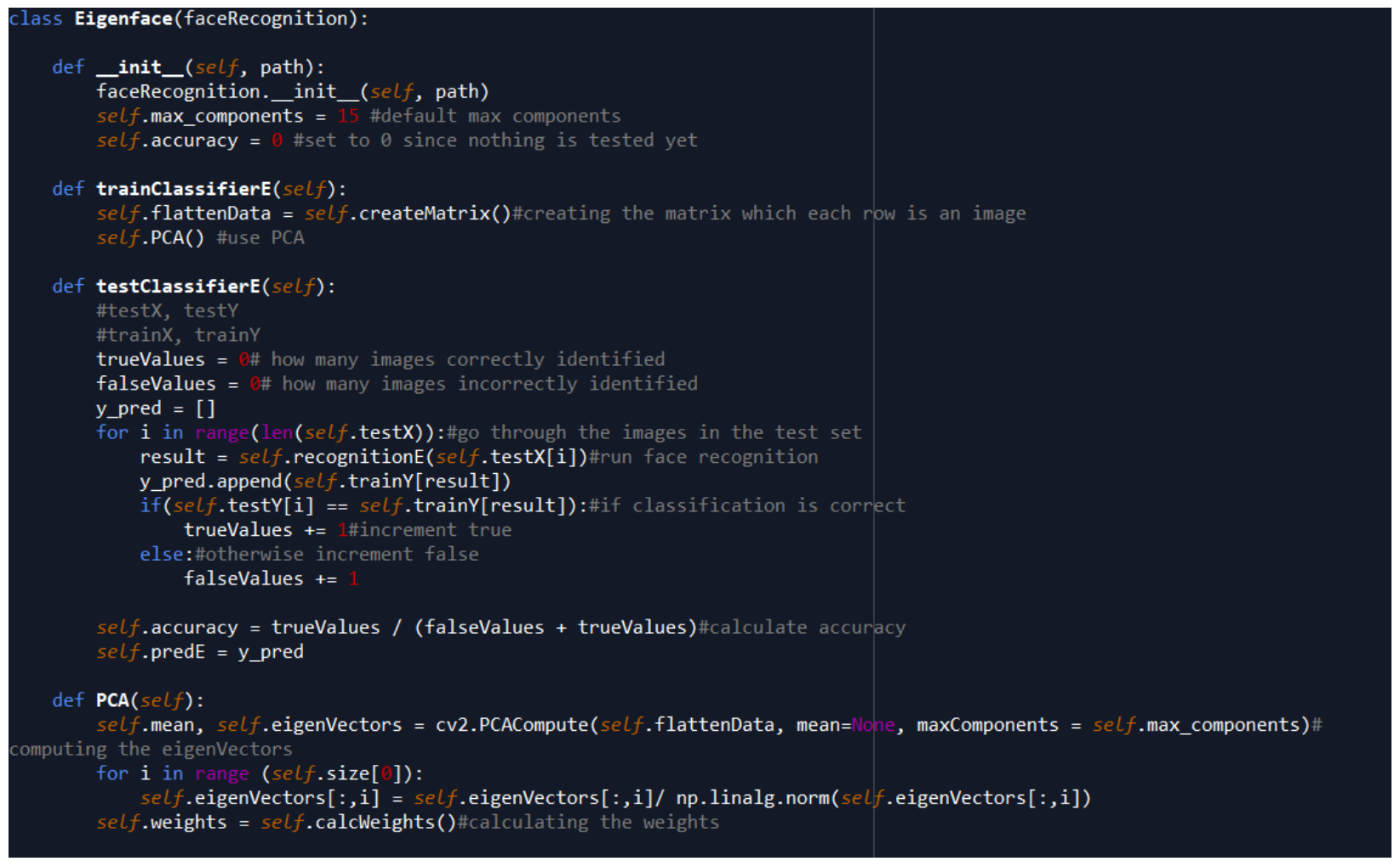




\section{Appendix D: Eigenfaces (Part 2)}

def recognitionE(self, inputFace):

\#Step 1: normalize face

inputFace $=$ inputFace. flatten()

normalizedFace $=$ inputFace - self.mean

normalizedFace $=$ normalizedFace.$T$

\#Step 2: project on eigenface (calculate the weights for input)

inputweights $=[]$

\#go through each eigenvector

for $i$ in range(len(self.eigenVectors)):

$w=s e l f \cdot$ eigenVectors [i] [:] $\operatorname{dot}($ normalizedFace)\#calculate weights inputWeights. append $(w)$

\#Step 3: Find Error either euclidean or mahalonbis

minValue $=9999999$ \#arbitrary high value

for $\mathrm{i}$ in range(len(self.weights)):

error $=\operatorname{self}$.euclideanDistance(inputWeights, self.weights[i])\#0 is temp position

if (error < minValue):

minValue $=$ error

location $=\mathrm{i}$

return location 
Appendix E: Fisherfaces (Part 1)

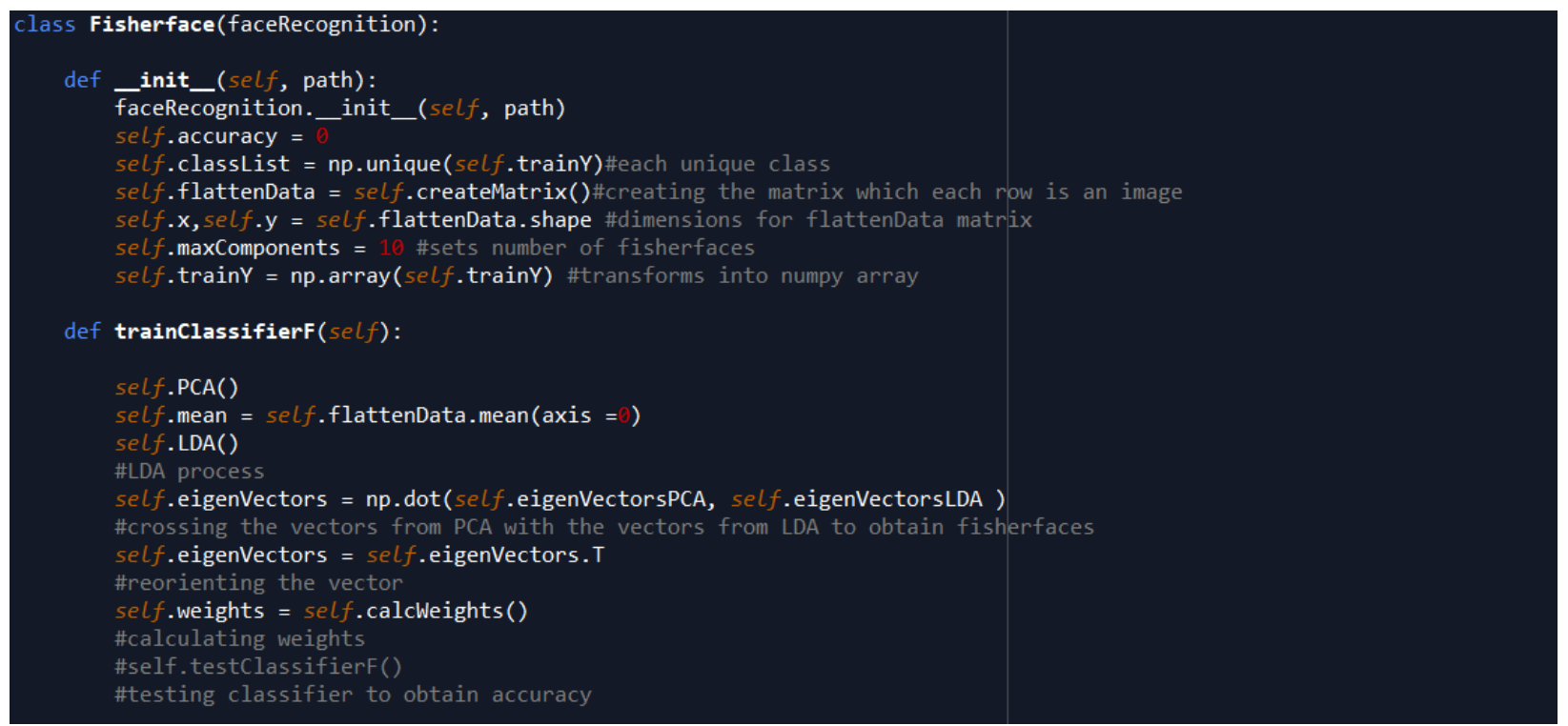




\section{Appendix F: Fisherfaces (Part 2)}

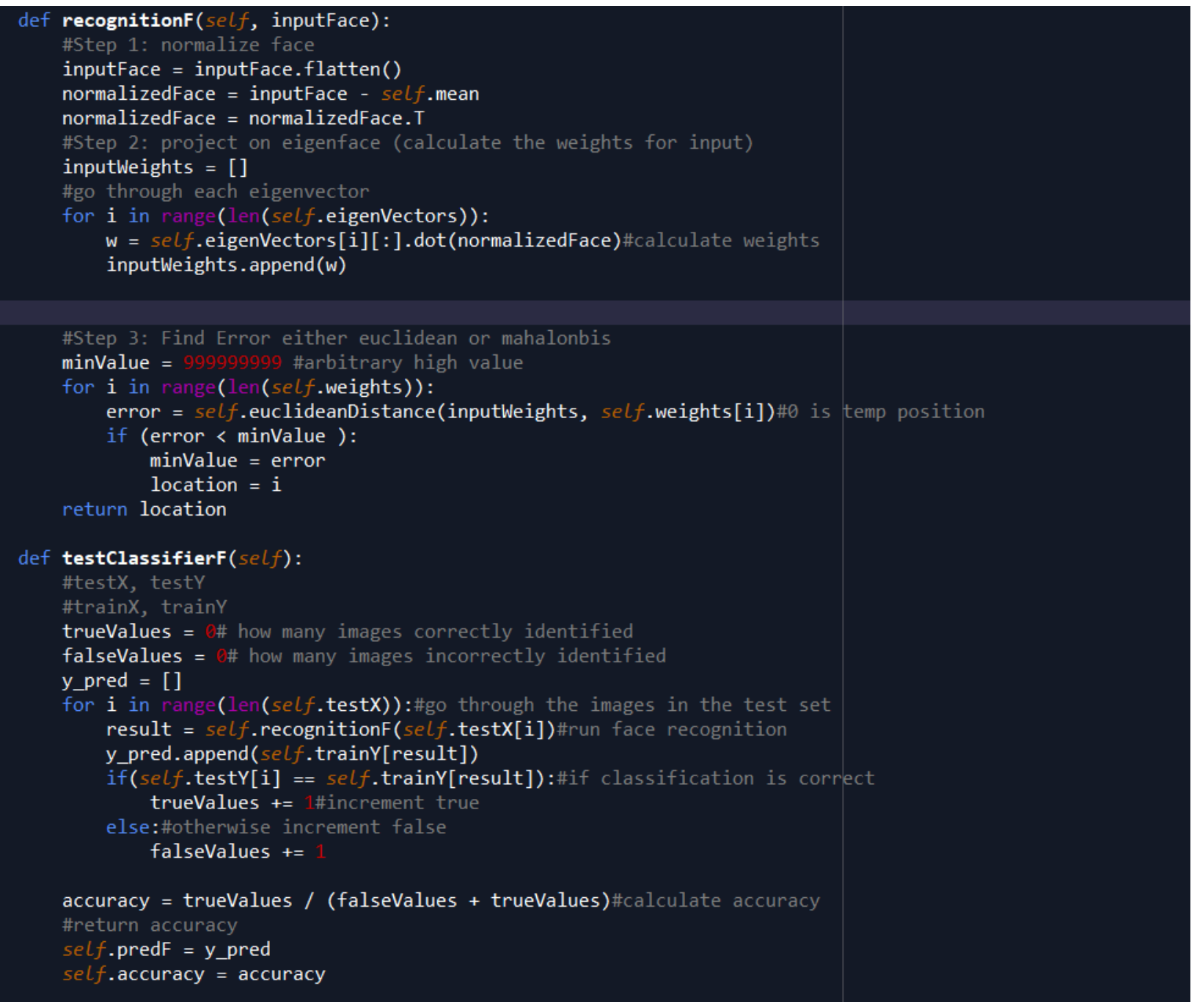




\section{Appendix G: Fisherfaces (Part 3)}

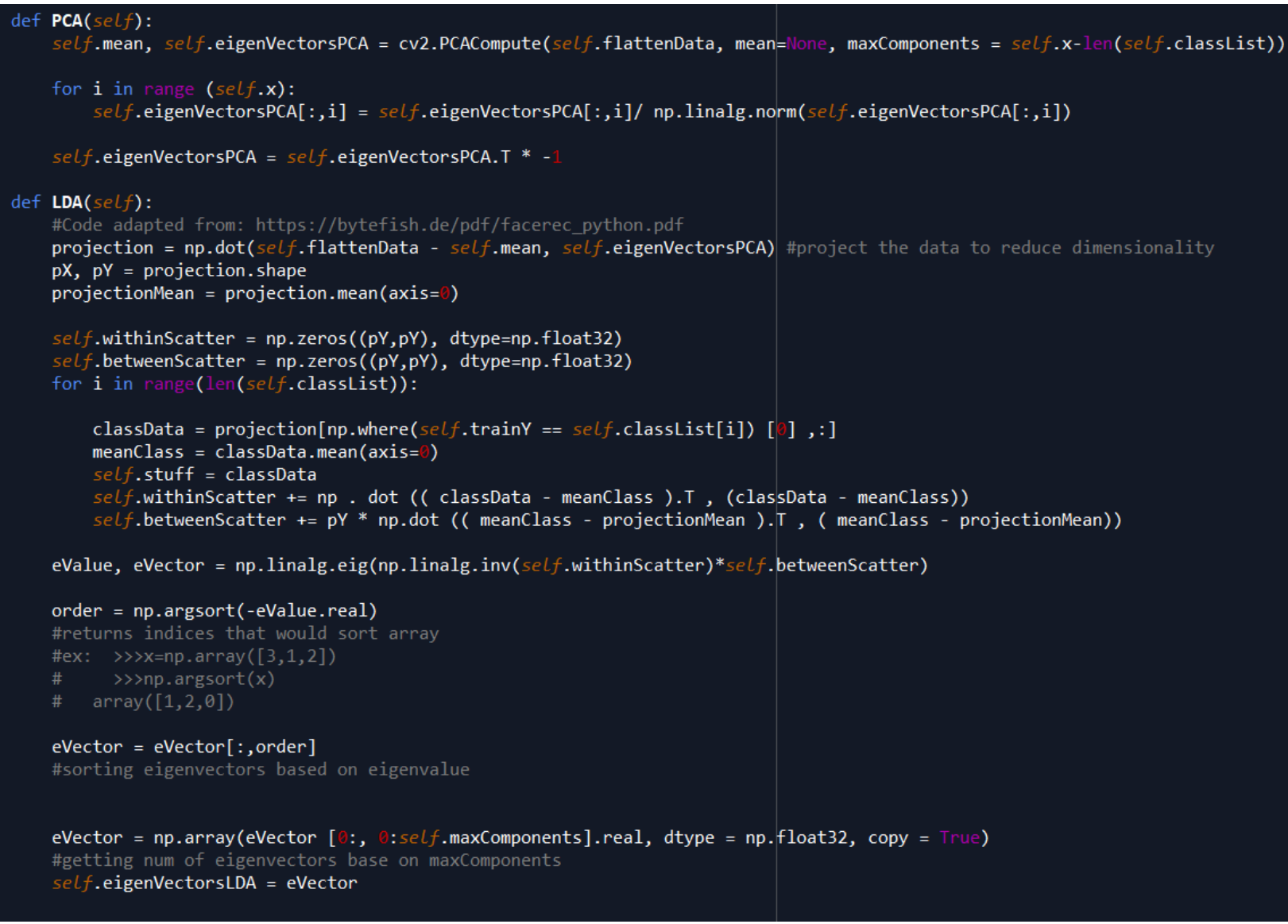




\section{Appendix H: Local Binary Patterns Histograms (Part 1)}

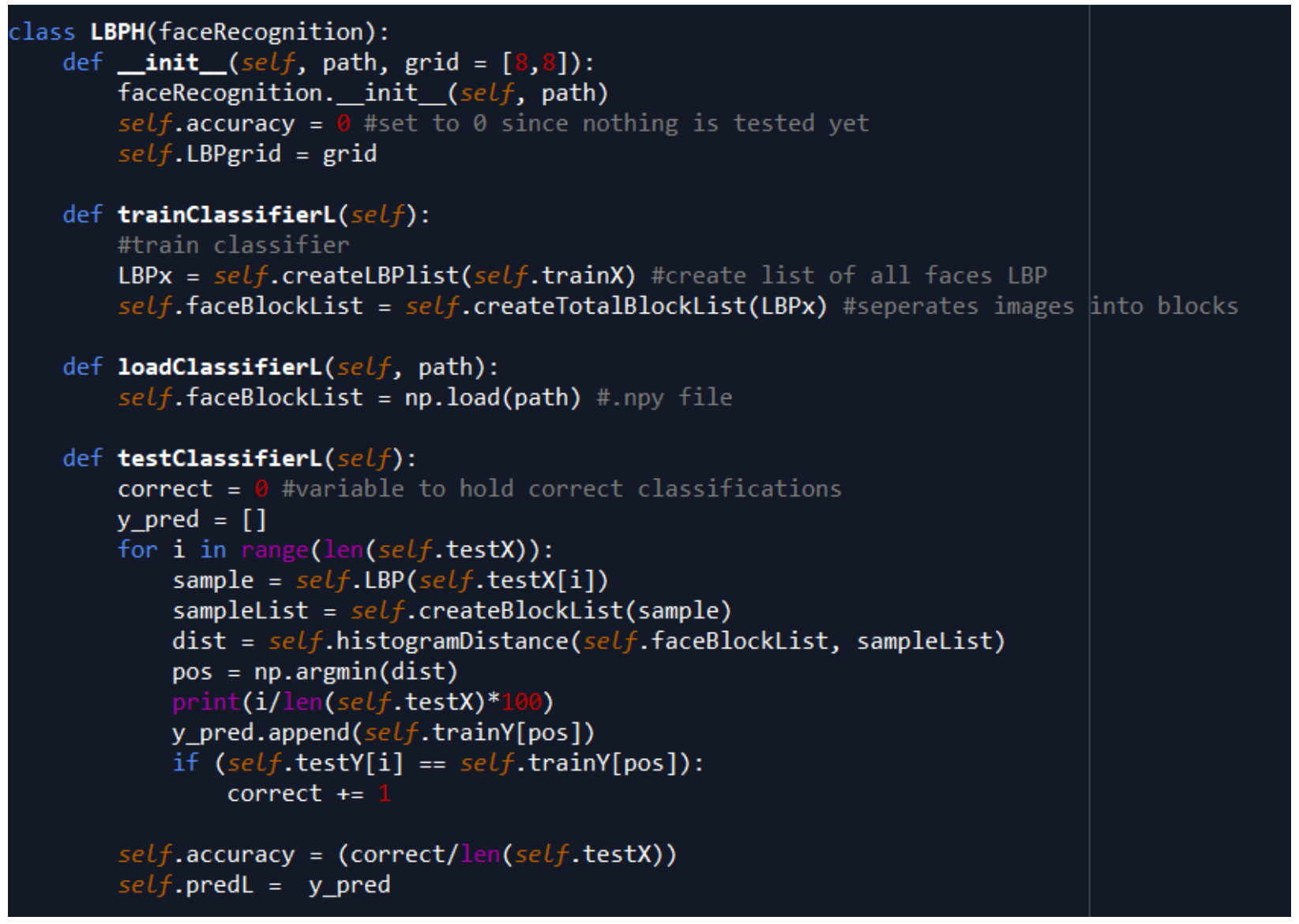




\section{Appendix I: Local Binary Patterns Histograms (Part 2)}

def createBlockList(self, inputImg):\#lbp img and grid size \#Create blocks for single img

blocksX = math. ceil(len(inputImg)/self.LBPgrid[0])

blocksY $=$ math. ceil $($ len $($ inputImg $[0]) / \operatorname{self} . \operatorname{LBPgrid}[1])$

\#create list of blocks from IMG

bList $=[]$

\#counter for $\mathrm{x}$

$\mathrm{cx}=$

for $\mathbf{i}$ in range(blocks $X)$ :

\#counter for $y$

cy $=$

for $\mathrm{j}$ in range(blocks $\mathrm{Y}$ ):

block = inputImg $[0+c x: 8+c x, 0+c y: 8+c y] \#$ collect the block

block = block.flatten ()$\# f$ latten the block

bList.append(block)\#add it to list

cy $+=8 \#$ increment by 8

cx $+=8$ \#increment by 8

return bList

def createTotalBlockList(self, inputList):

\#create BlockList for every image

facesBlockList $=[]$

for $i$ in range(len(inputList)):

block $=$ self. . createBlockList (inputList $[i])$

facesBlockList.append(block)

return facesBlockList 


\section{Appendix J: Local Binary Patterns Histograms (Part 3)}

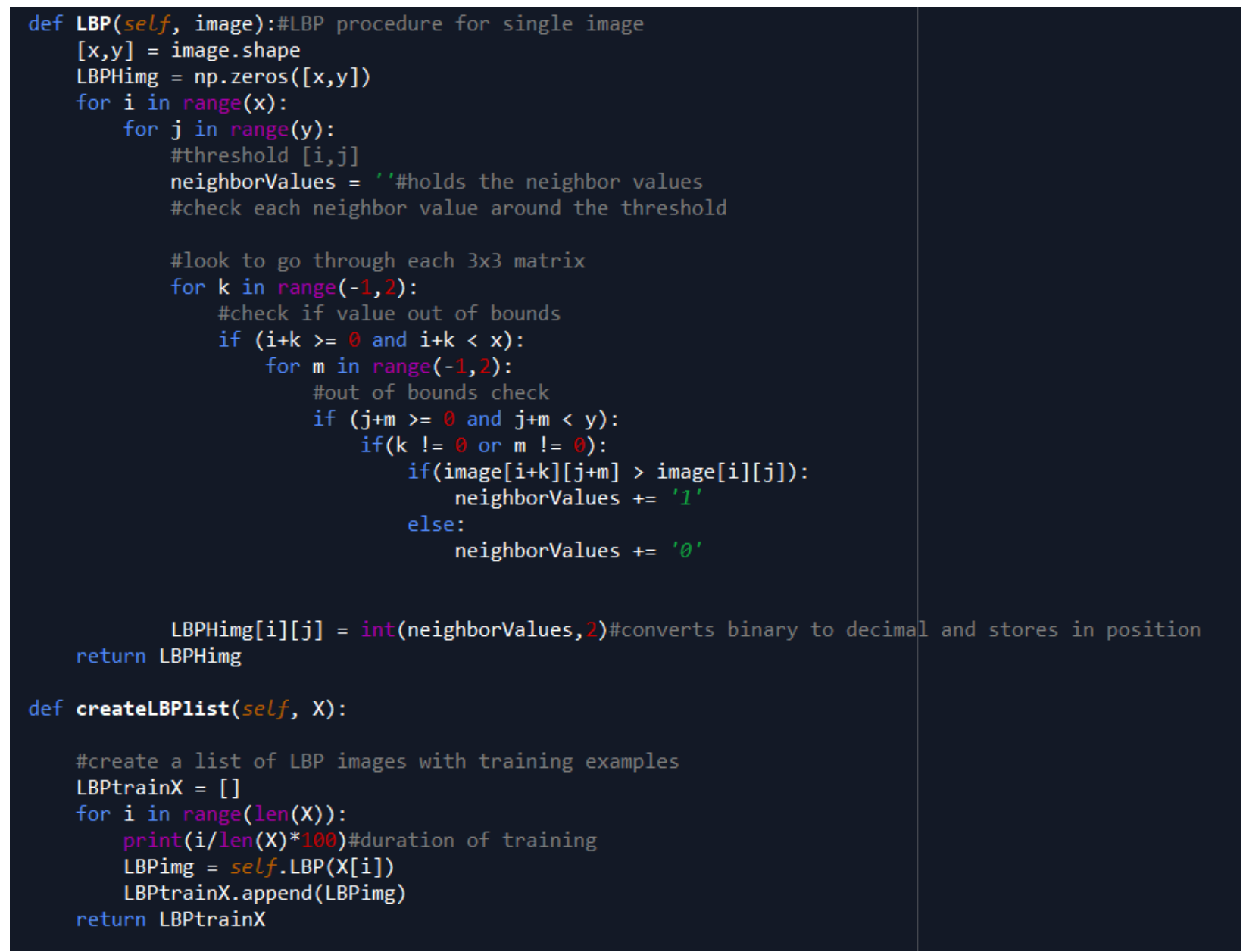




\section{Appendix K: Local Binary Patterns Histograms (Part 4)}

def histogramDistance(self, facelist, faceTest):\#check distance between histograms \#facelist - list of blocks for each face in training sample \#faceTest - face block list that is being tested \#collect all the distances from sample image allDistance $=[]$ \#go through all faces in list for $j$ in range(len (facelist)): xdistance $=[]$ \#distance for each face

for $i$ in range(len(faceTest)): distance $=$ self.euclideanDistance $($ faceTest $[i]$,faceList $[j][i])$ xdistance.append(distance)\#get the distance

allDistance. append(sum(xdistance)) 
Appendix L: EFL Hybrid Algorithm

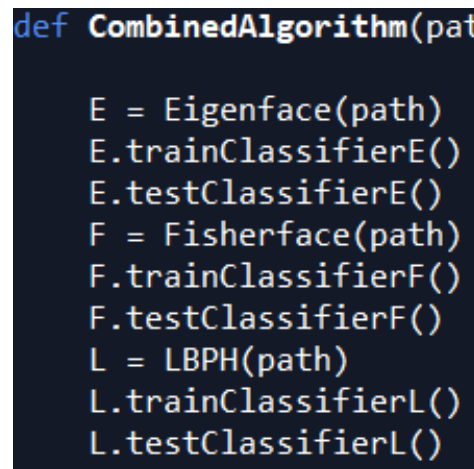

\#compare results

LBPHc_pred $=[]$

for $i$ in range(len(E.predE)):

if $(E \cdot p r e d E[i]==F \cdot \operatorname{predF}[i])$ :

LBPHc_pred.append (F.predF [i])

else:

LBPHc_pred.append(L.predL[i])

\#calculate accuracy

correct $=$

for $i$ in range(Ien(LBPHc_pred)):

if (LBPHc_pred[i] == L.testY[i]):

correct $+=$

LBPHc_acc $=$ correct/len (LBPHc_pred)

return LBPHc_acc, LBPHc_pred 